\title{
Copper-Catalyzed Conjugate Additions to Isocyanoalkenes
}

John-Paul R. Marrazzo, Allen Chao, Yajun Li, and Fraser F. Fleming*

Department of Chemistry, Drexel University, 3141 Chestnut St., Philadelphia PA 19104

* fleming@drexel.edu

\section{Supporting Information}

\section{Table of Contents}

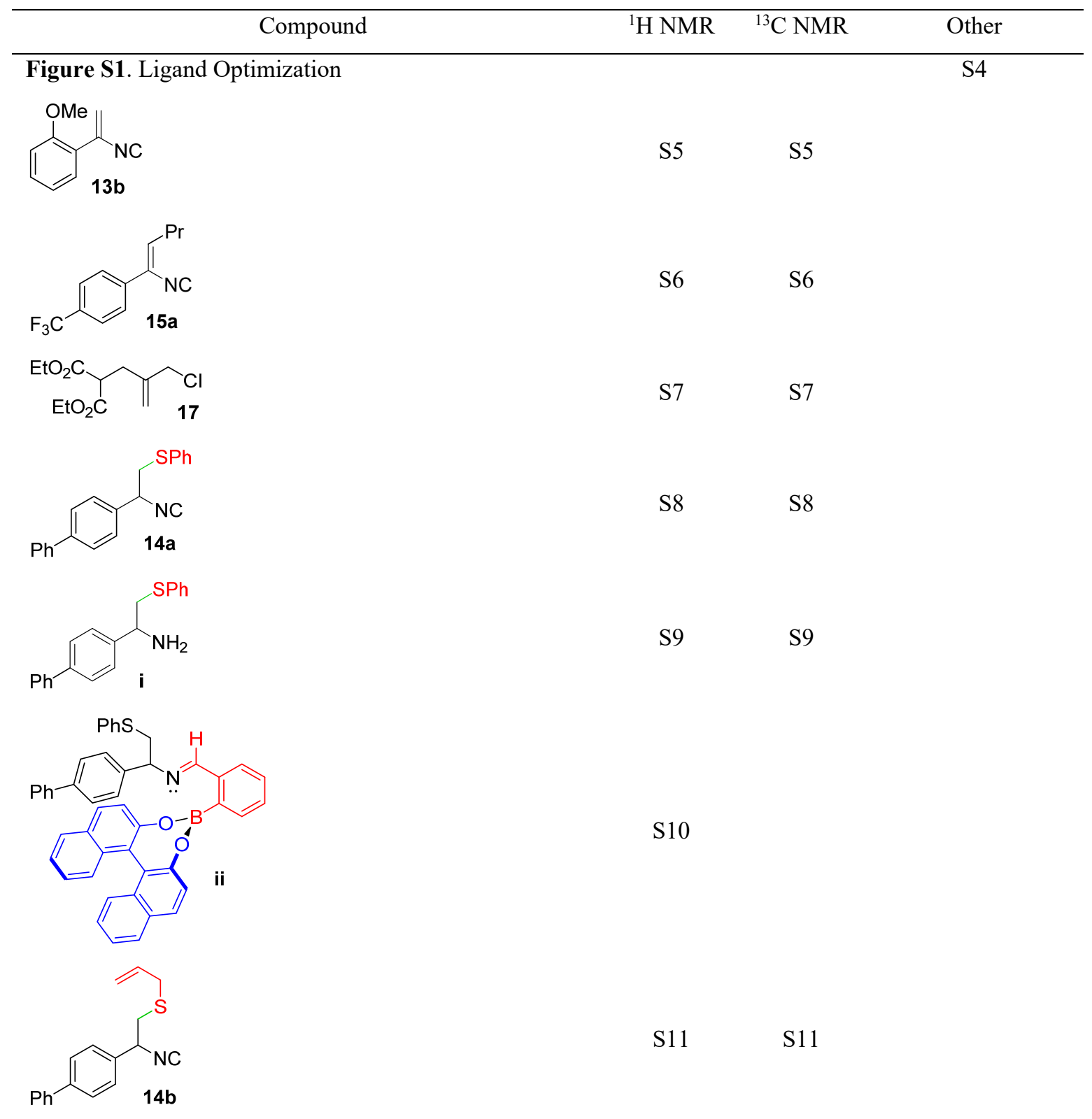



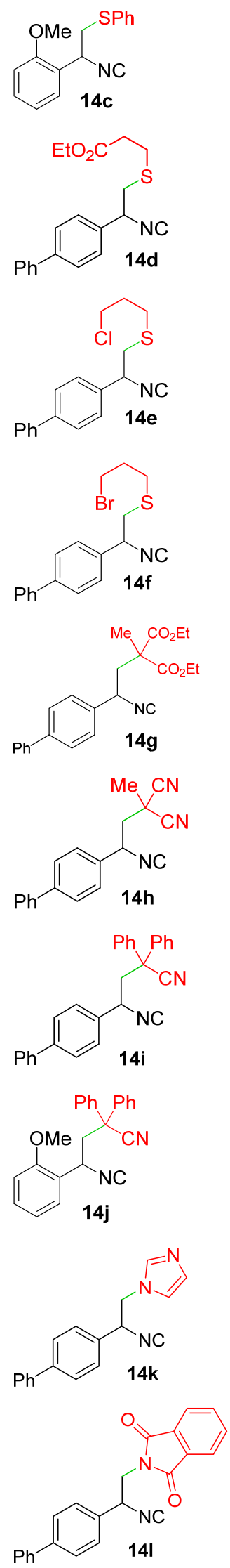

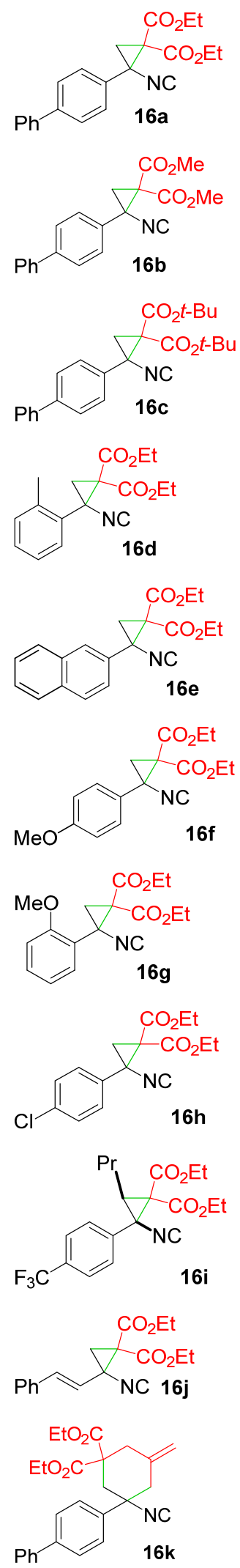

${ }^{19} \mathrm{~F}$ NMR - S31

NOESY - S31
Figure S2.

Chromatogram

S35 


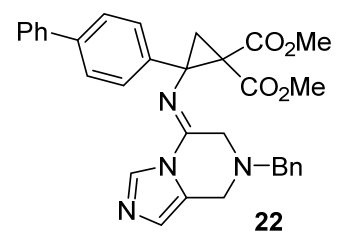

Figure S3. Suppression of downfield ${ }^{13} \mathrm{C}$ NMR resonances on addition of Cu(I)I to 13a. $\quad$ S36

References

Figure S1. Ligands Evaluated in the Conjugate Addition of Nucleophiles to 13a.
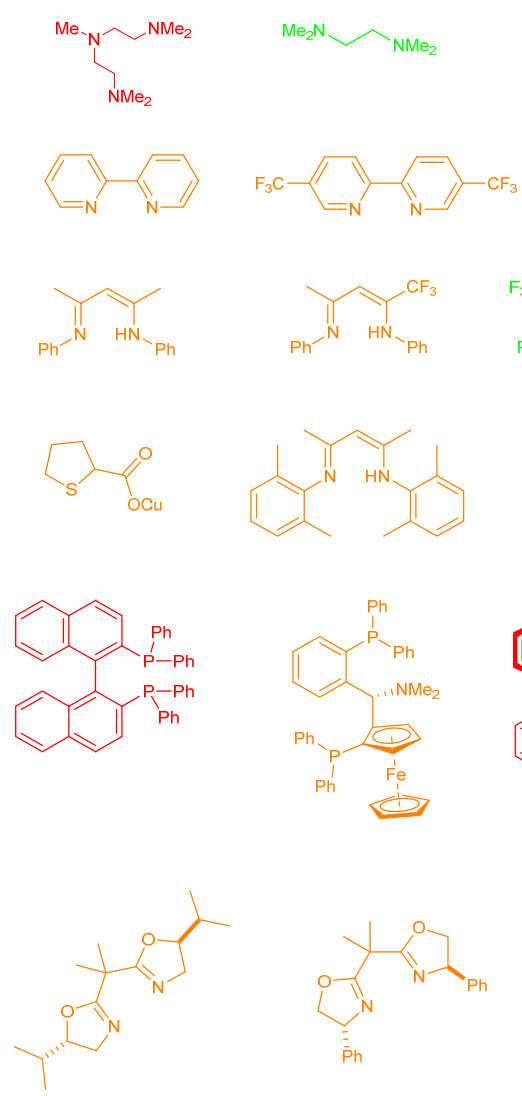

Red $=$ No conjugate addition Orange $=$ Some conjugate addition Green = Clean conjugate addition"

* Based on a qualitative analysis of the $1 \mathrm{H}$ NMR of the crude reaction mixture 

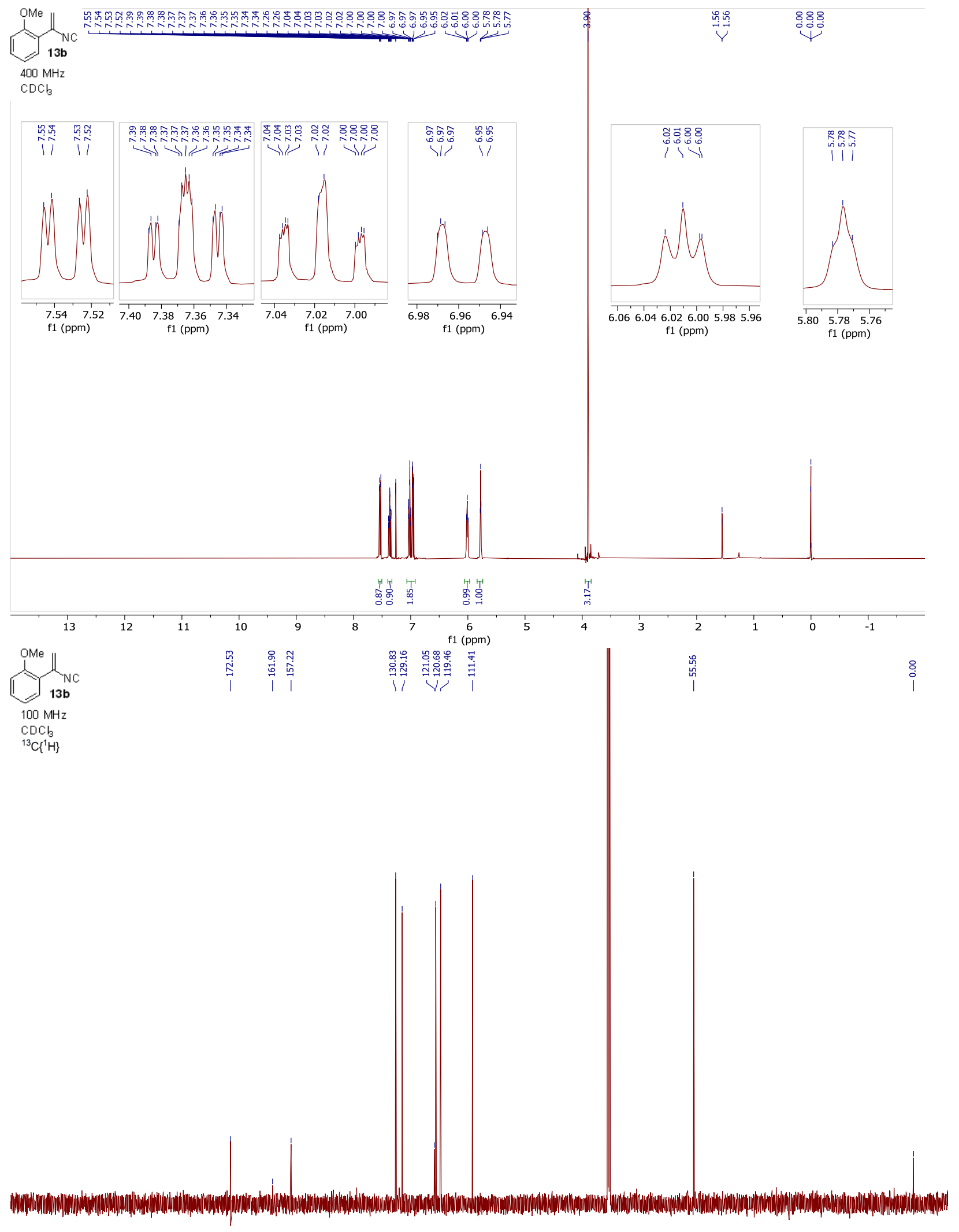

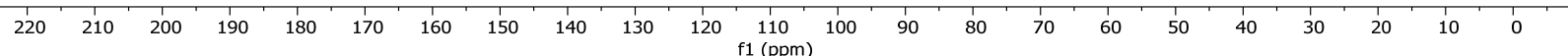




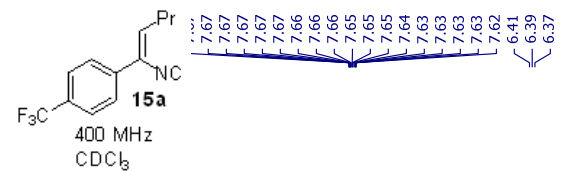

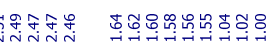
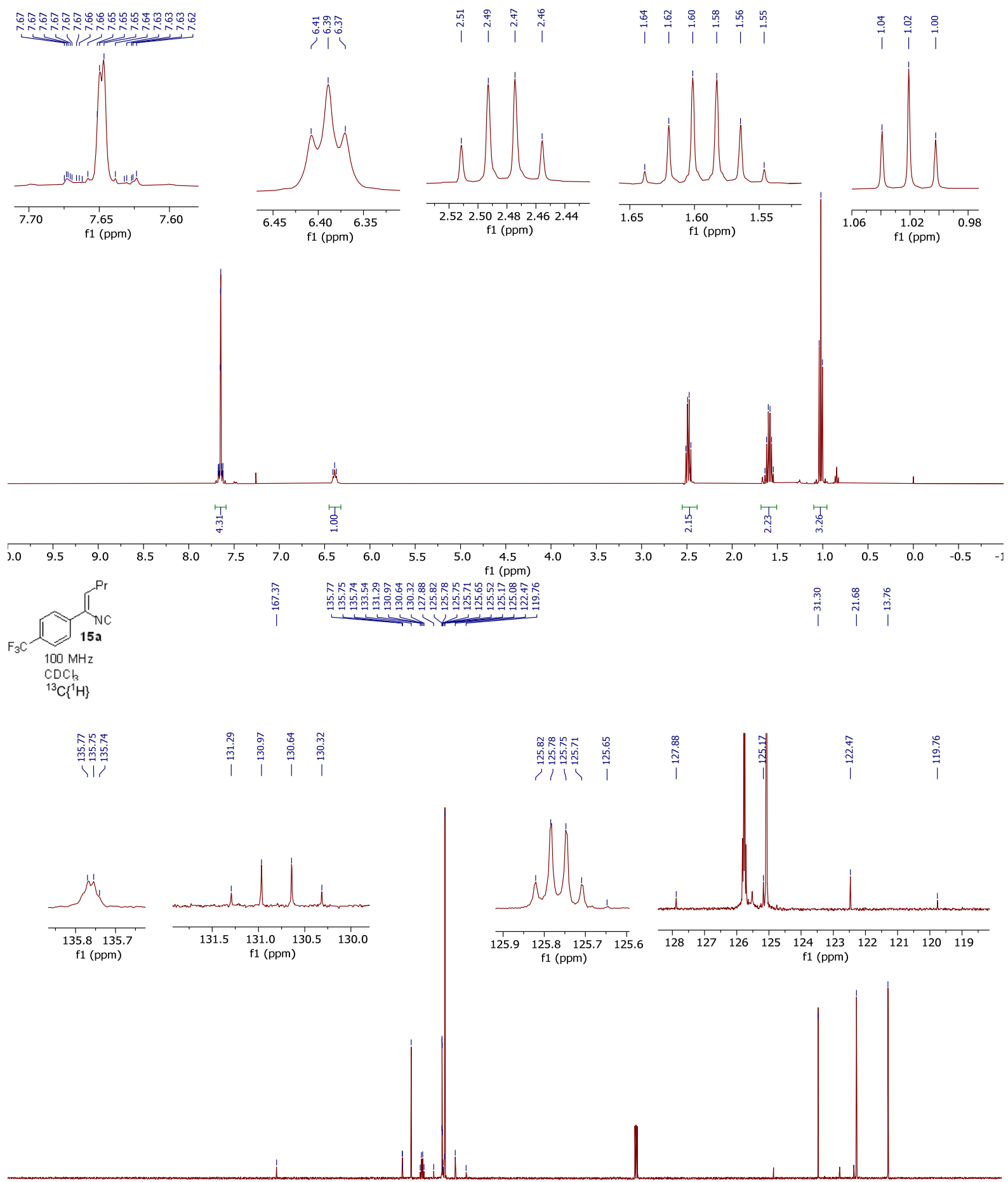

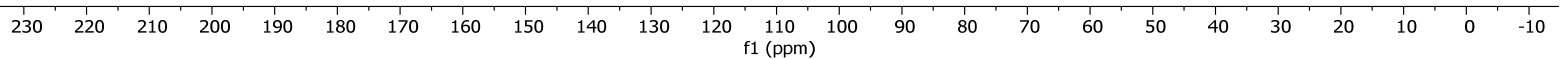




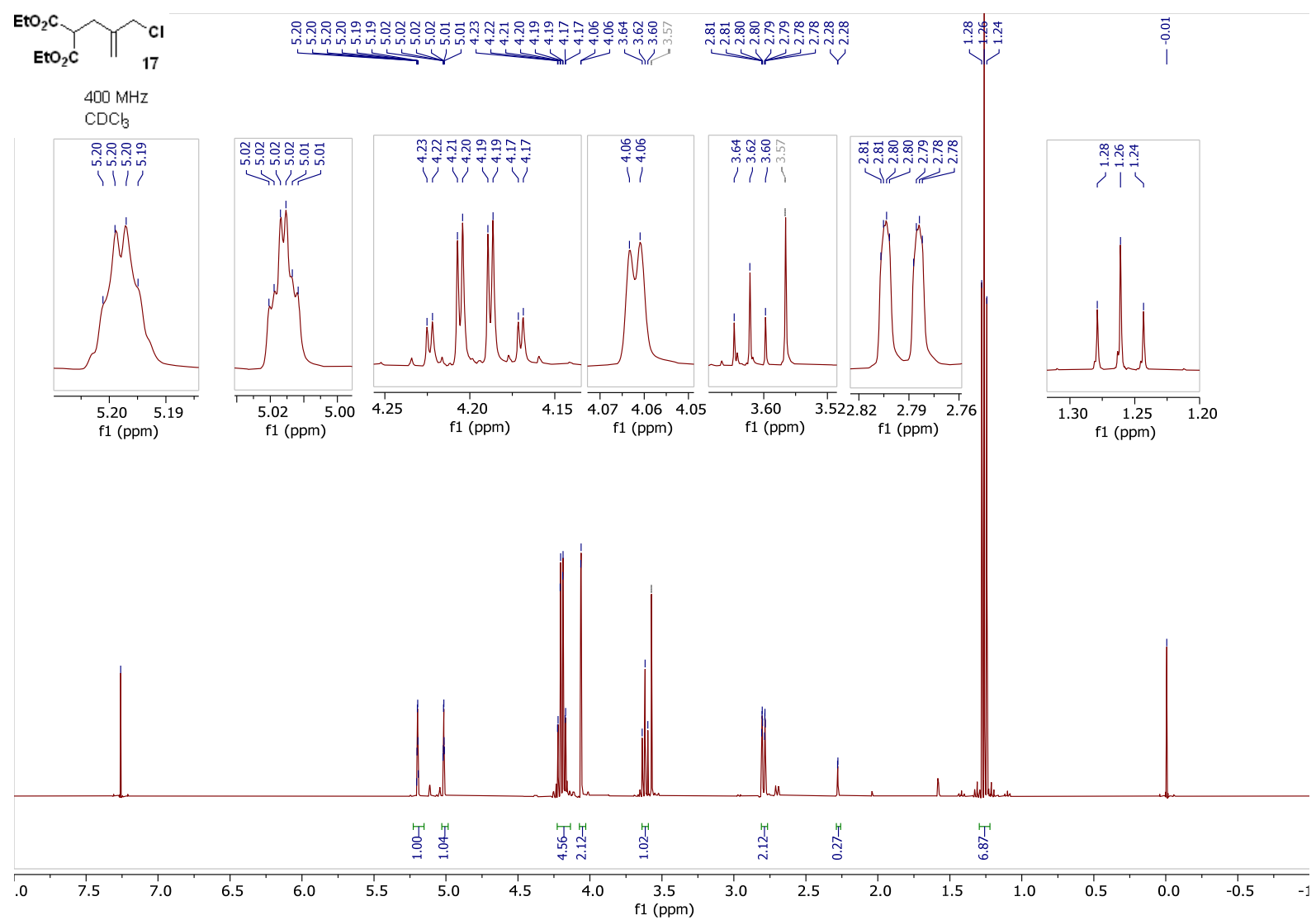

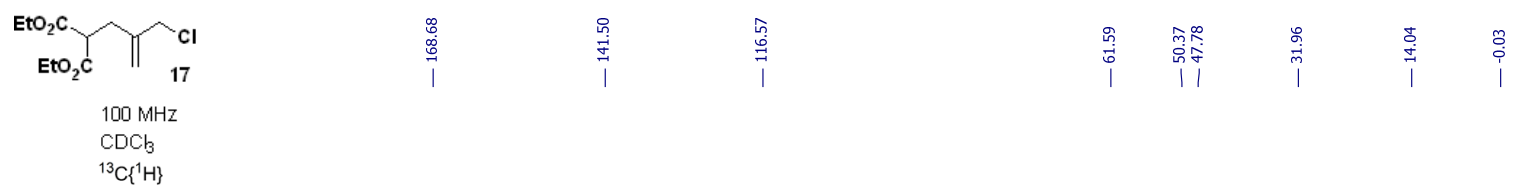

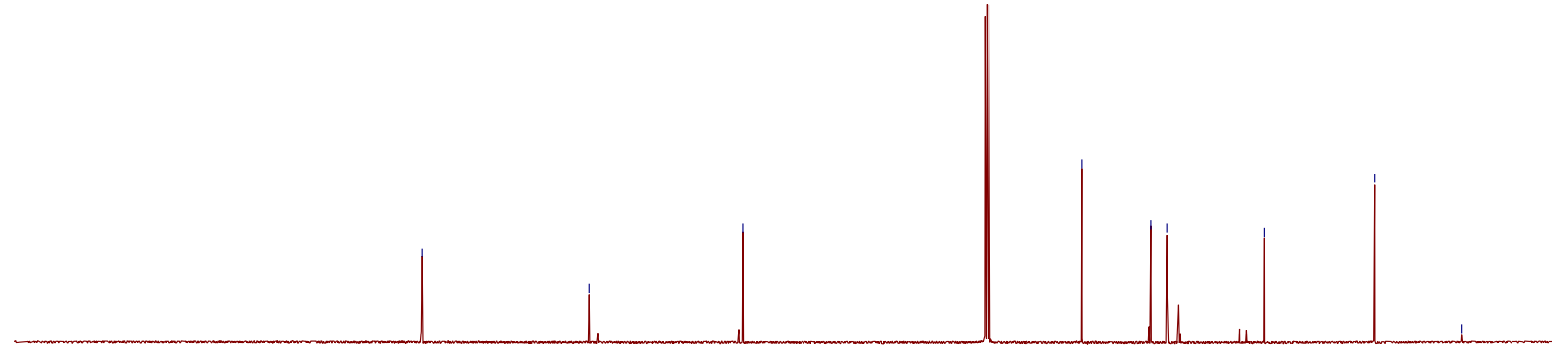

$\begin{array}{lllllllllllllllllllllllllllllll}230 & 220 & 210 & 200 & 190 & 180 & 170 & 160 & 150 & 140 & 130 & 120 & 110 & 100 & 90 & 80 & 70 & 60 & 50 & 40 & 30 & 20 & 10 & 0 & -10\end{array}$ 

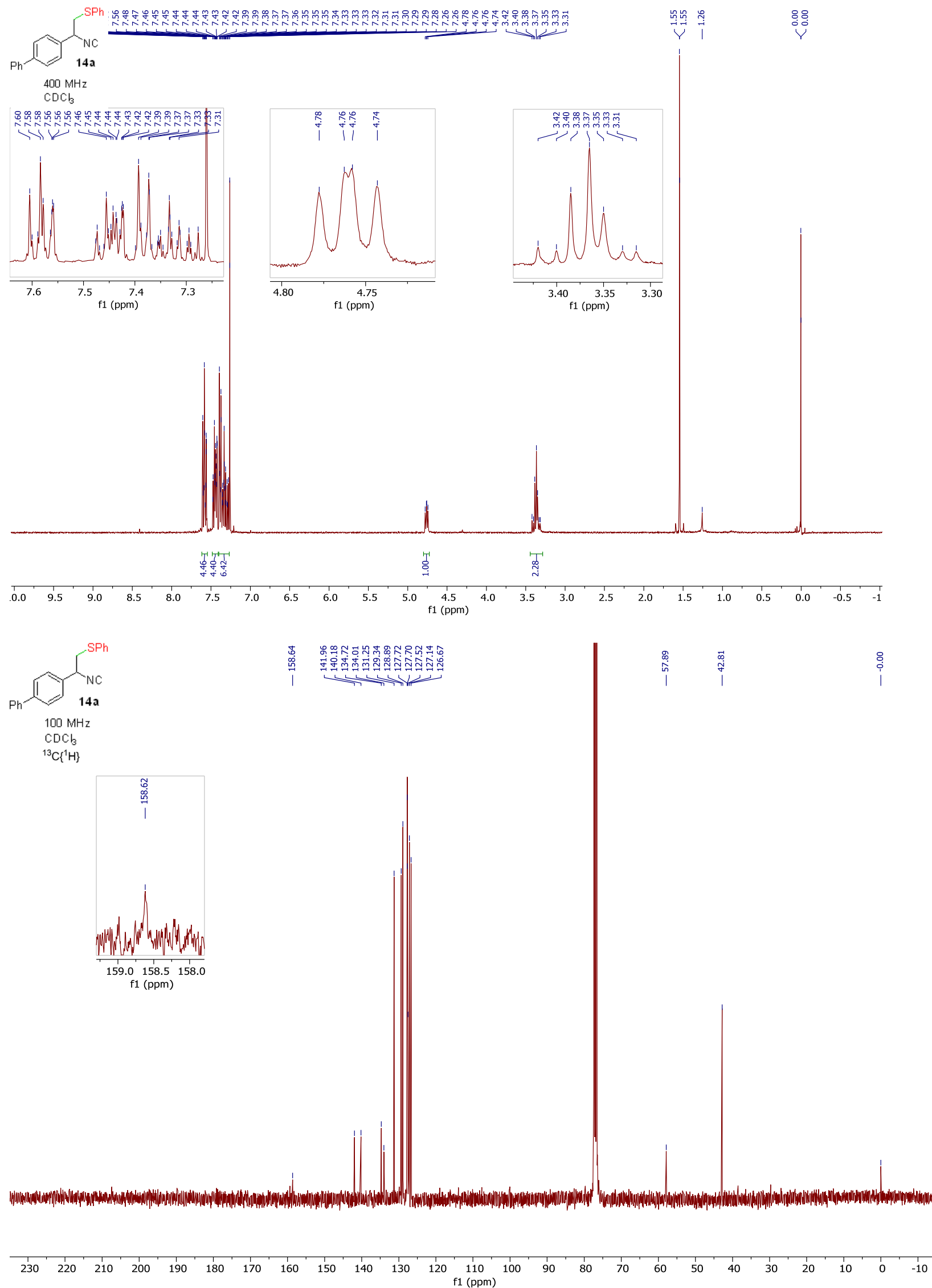

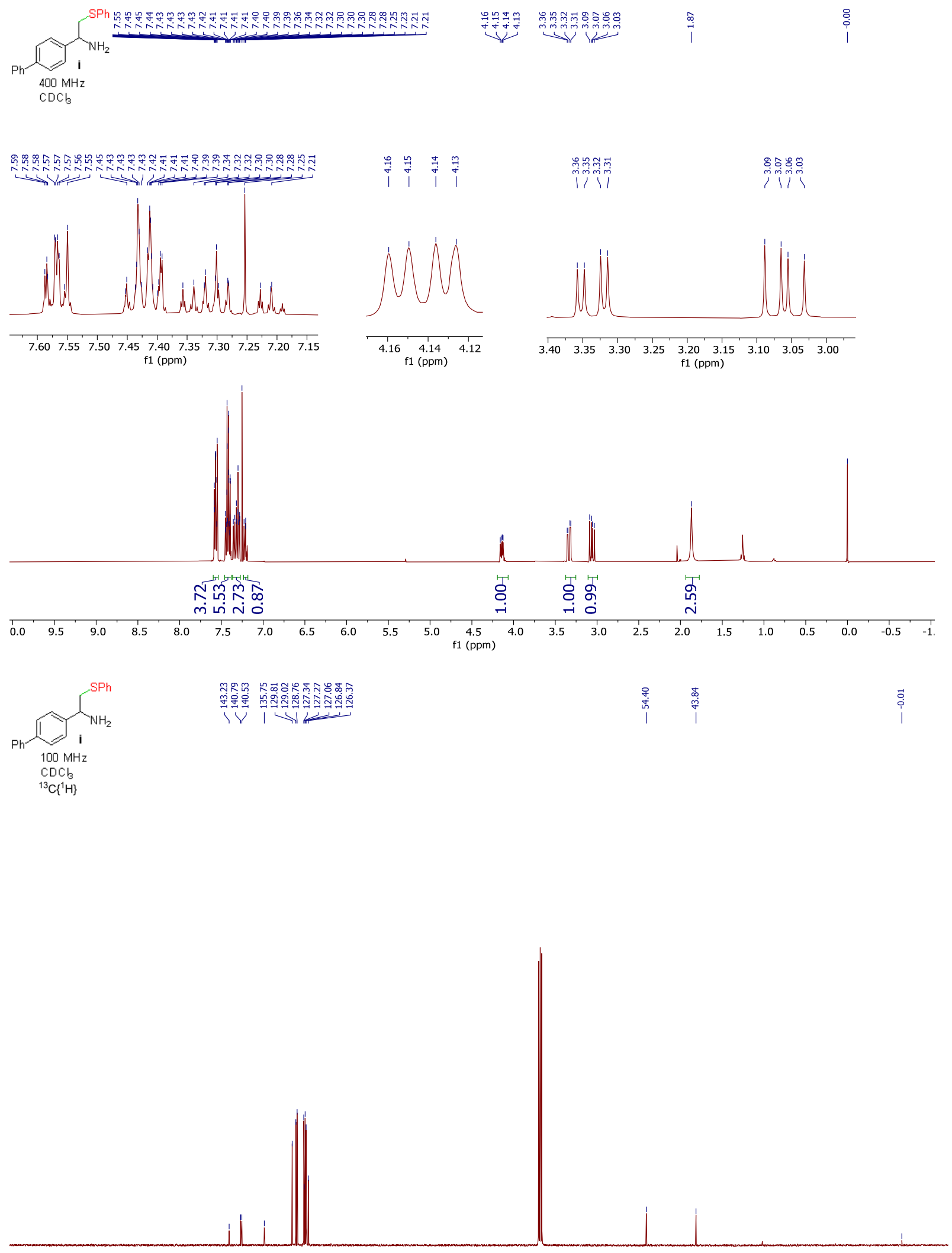

\begin{tabular}{llllllllllllllllllll}
\hline 90 & 180 & 170 & 160 & 150 & 140 & 130 & 120 & 110 & 100 & 90 & 80 & 70 & 60 & 50 & 40 & 30 & 20 & 10 & 0
\end{tabular} 


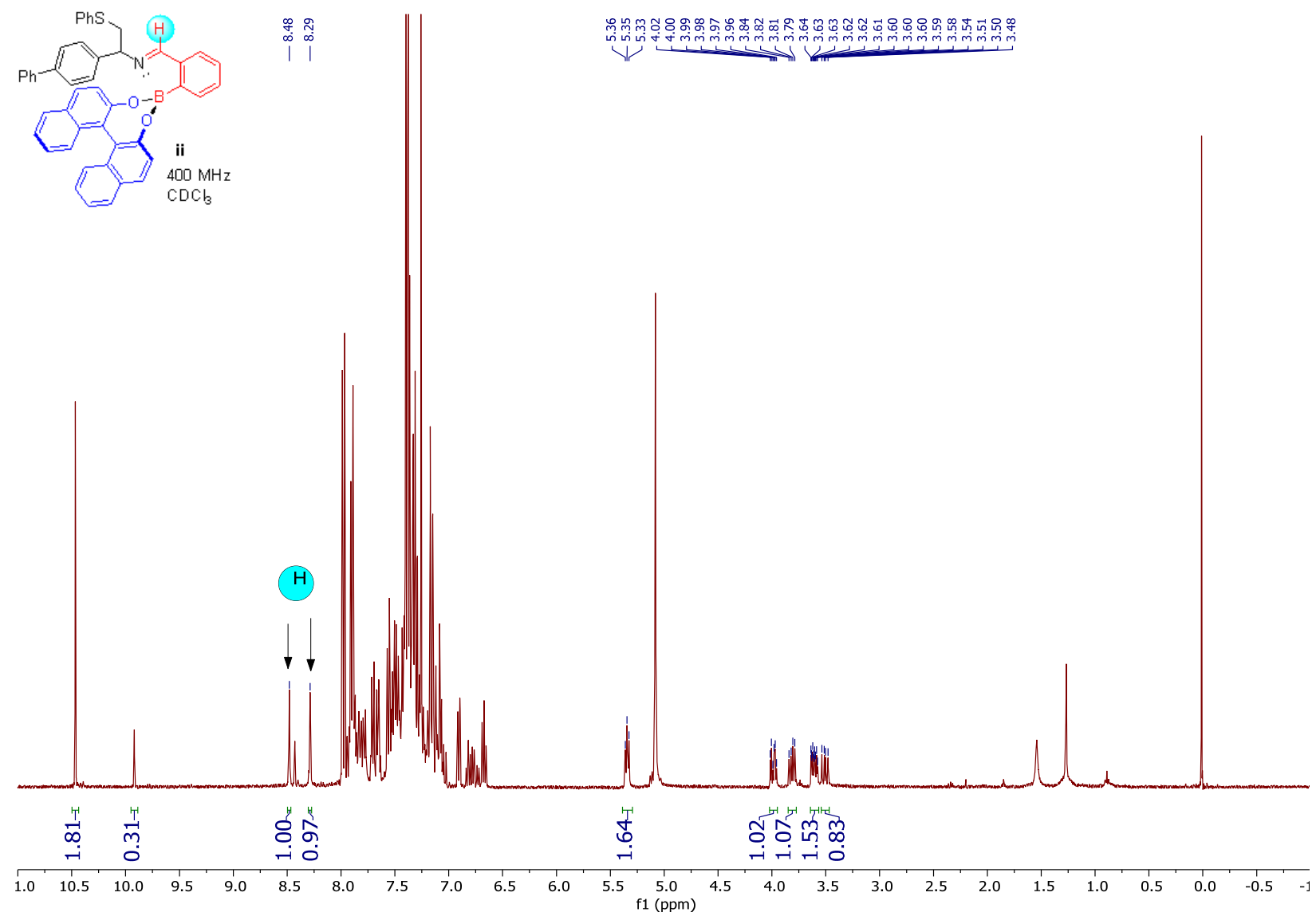



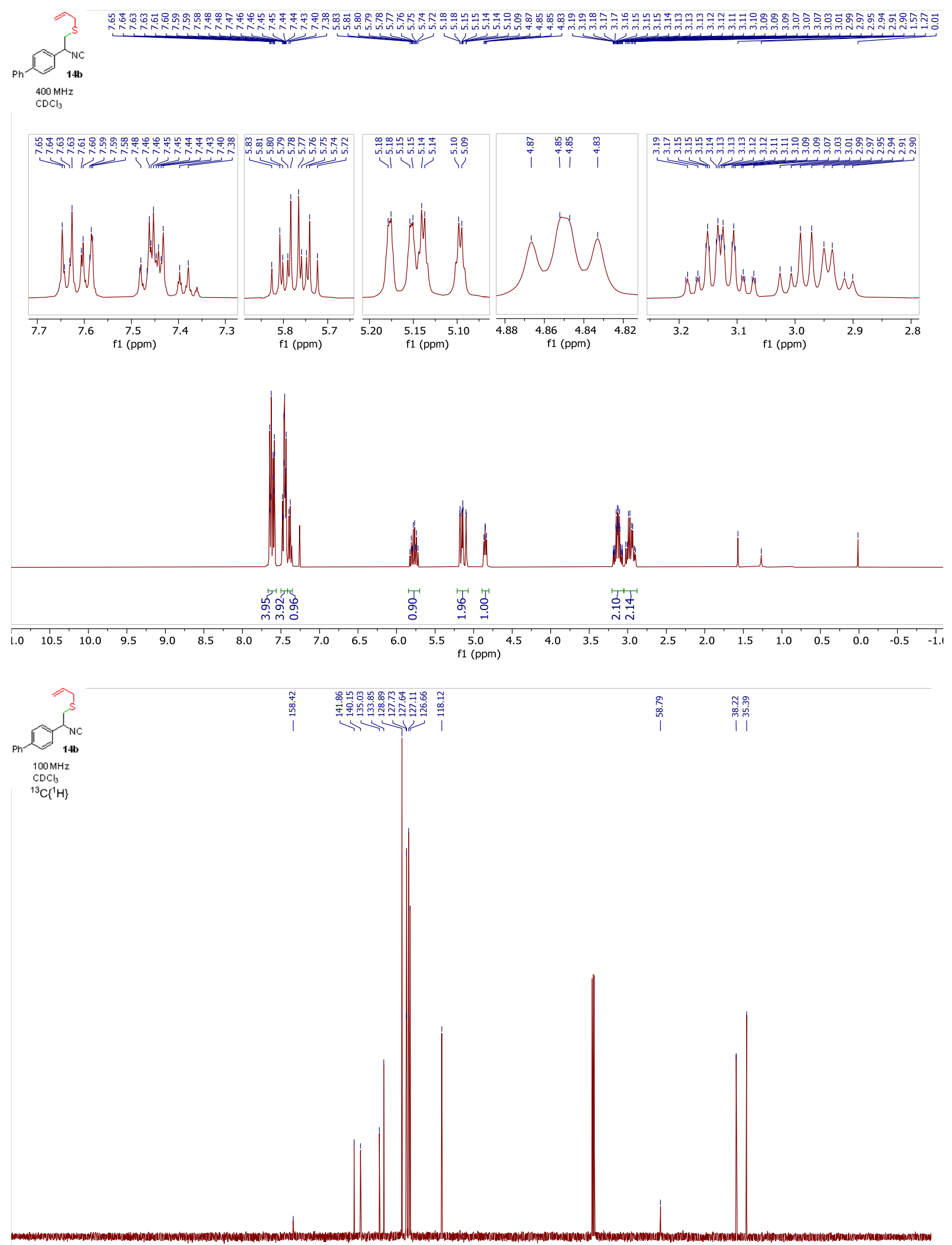

$\begin{array}{lllllllllllllllllllllllllllll}230 & 220 & 210 & 200 & 190 & 180 & 170 & 160 & 150 & 140 & 130 & 120 & 110 & 100 & 90 & 80 & 70 & 60 & 50 & 40 & 30 & 20 & 10 & 0 & -10\end{array}$ 

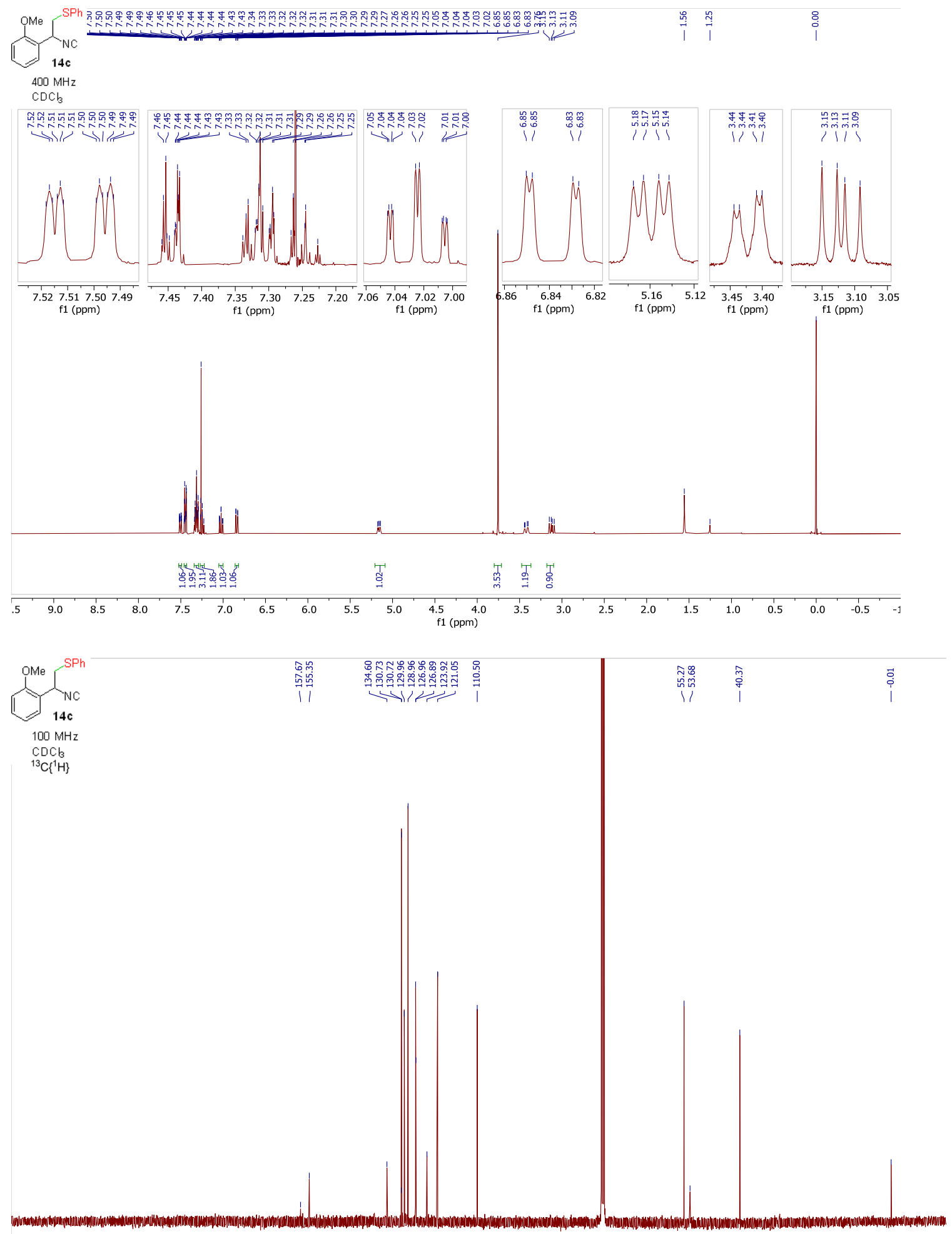

\begin{tabular}{|l|lllllllllllllllllllllllllll}
\hline & 130 & 220 & 210 & 200 & 190 & 180 & 170 & 160 & 150 & 140 & 130 & 120 & 110 & 100 & 90 & 80 & 70 & 60 & 50 & 40 & 30 & 20 & 10 & 0 & -10
\end{tabular} 

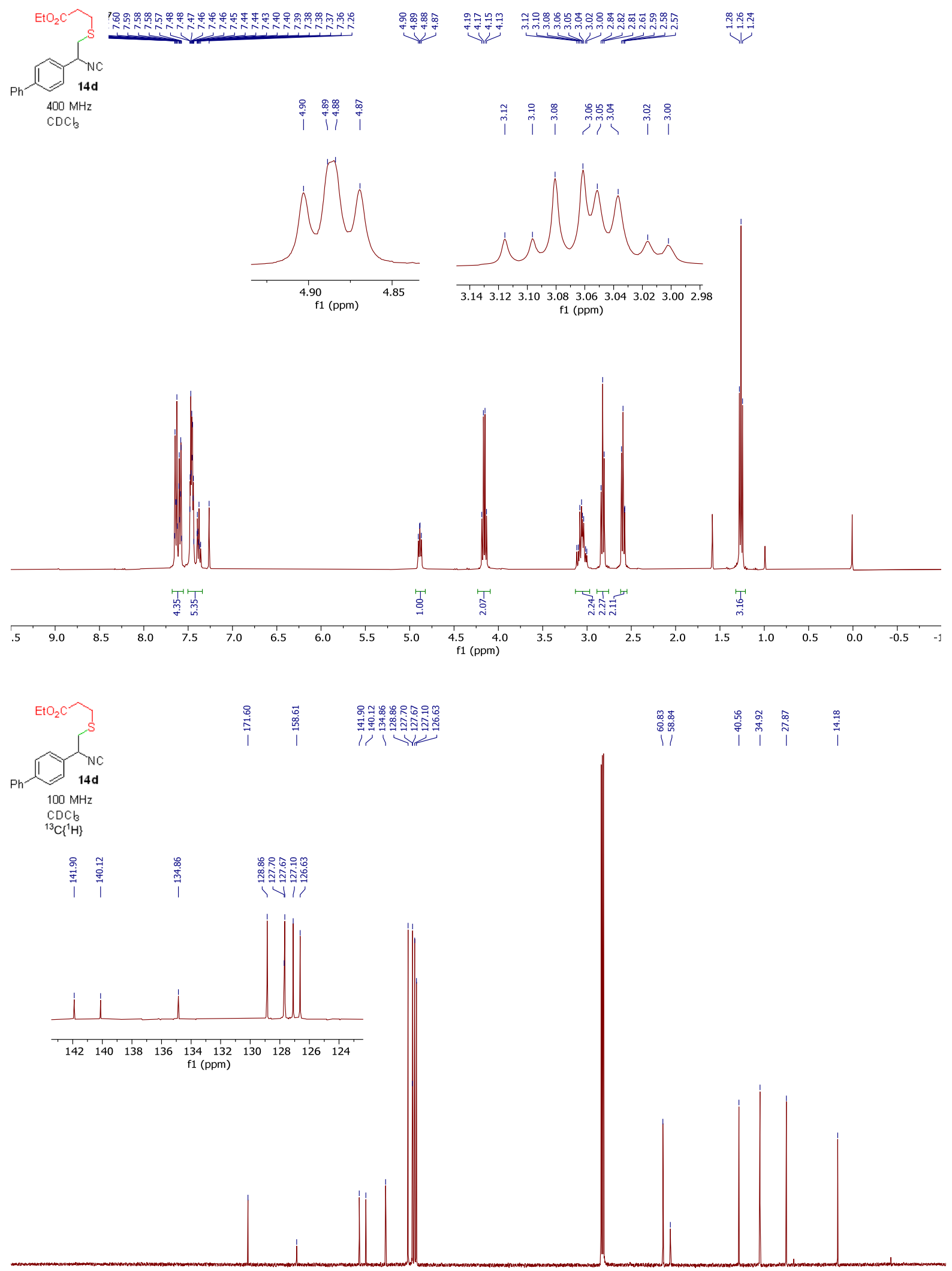

$\begin{array}{llllllllllllllllllllllllllllllllllll}230 & 220 & 210 & 200 & 190 & 180 & 170 & 160 & 150 & 140 & 130 & 120 & 110 & 100 & 90 & 80 & 70 & 60 & 50 & 40 & 30 & 20 & 10 & 0 & -10\end{array}$ 

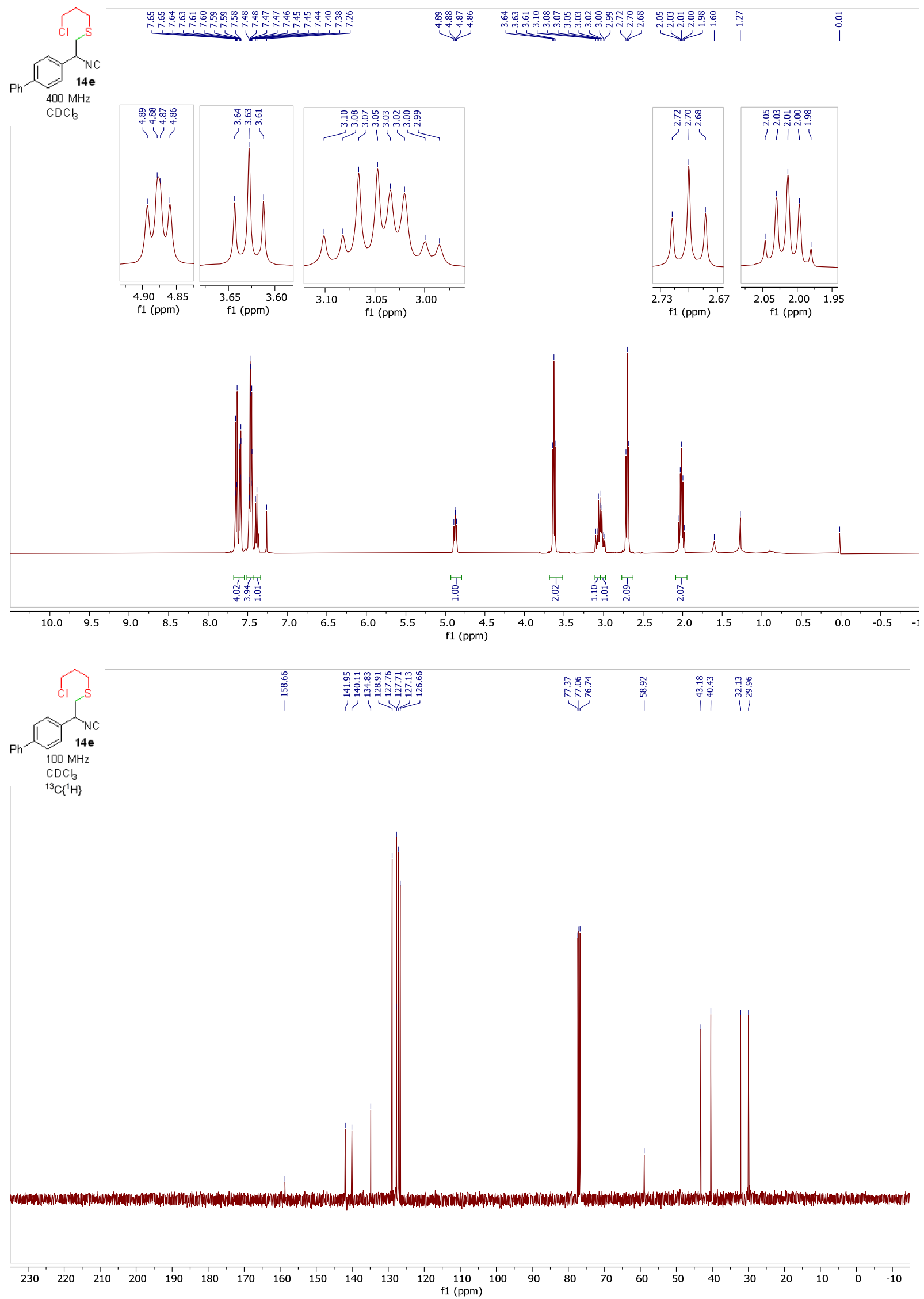

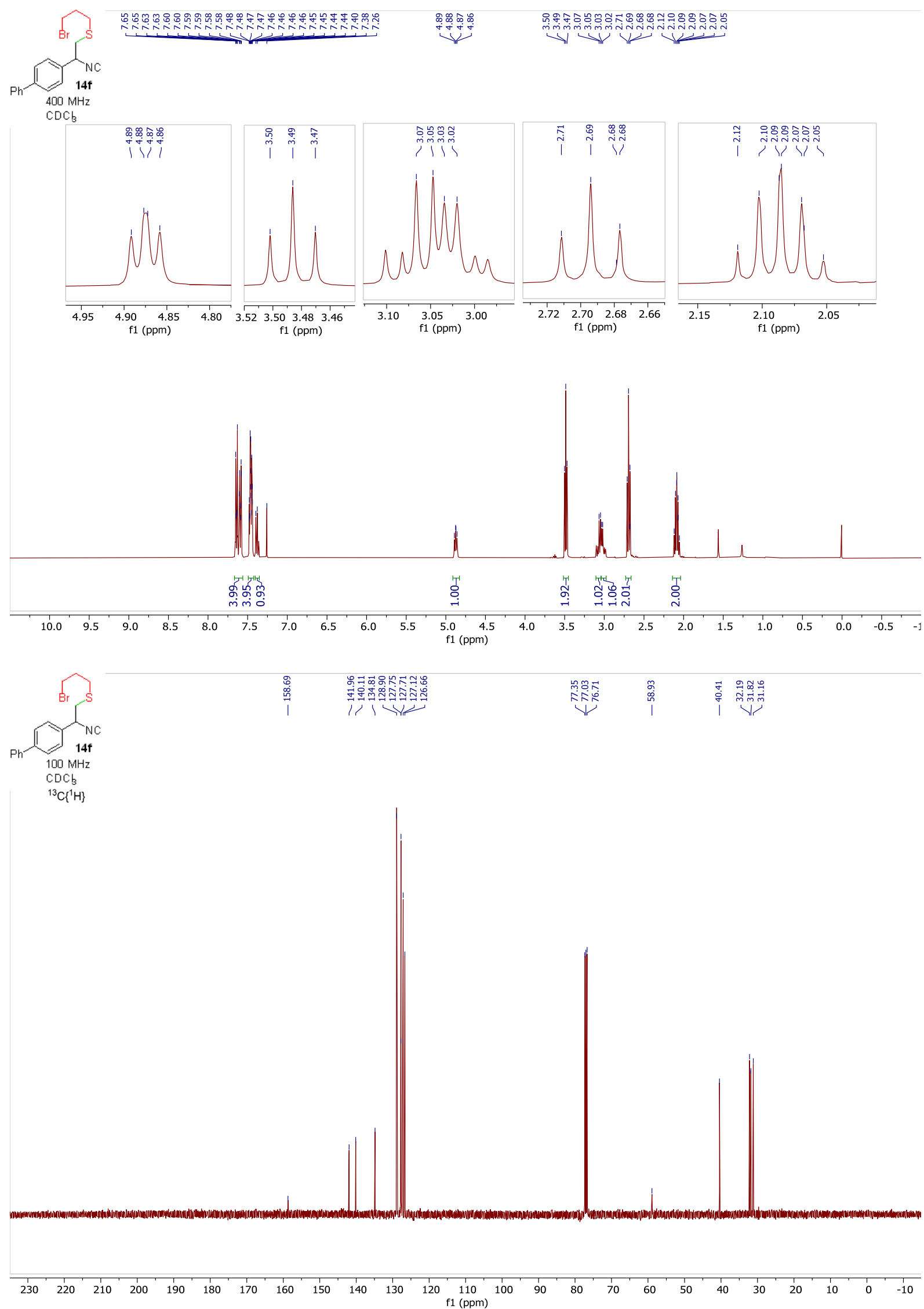

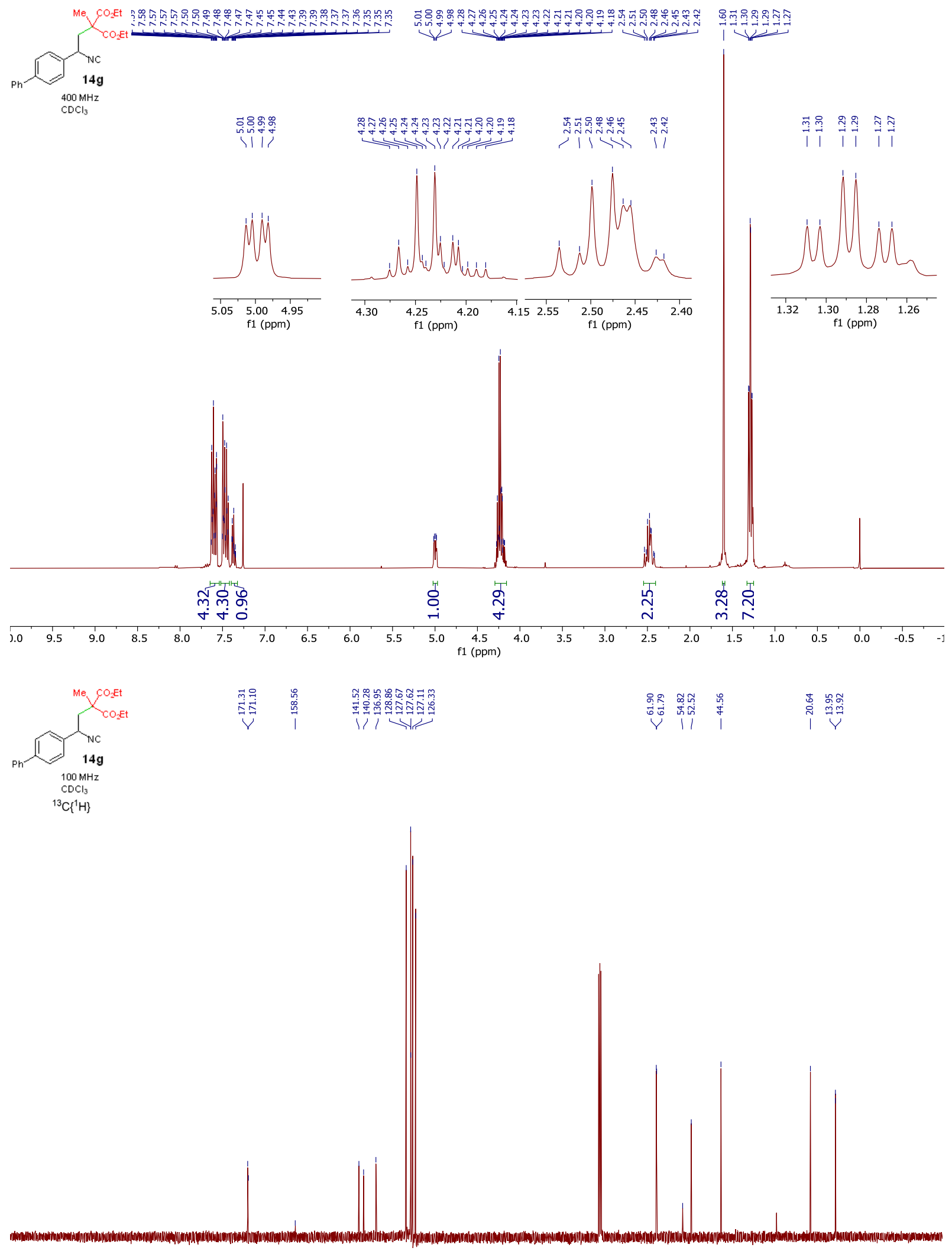

$\begin{array}{lllllllllllllllllllllllllll}230 & 220 & 210 & 200 & 190 & 180 & 170 & 160 & 150 & 140 & 130 & 120 & \begin{array}{c}110 \\ \mathrm{f} 1(\mathrm{ppm})\end{array} & 100 & 90 & 80 & 70 & 60 & 50 & 40 & 30 & 20 & 10 & 0 & -10\end{array}$ 

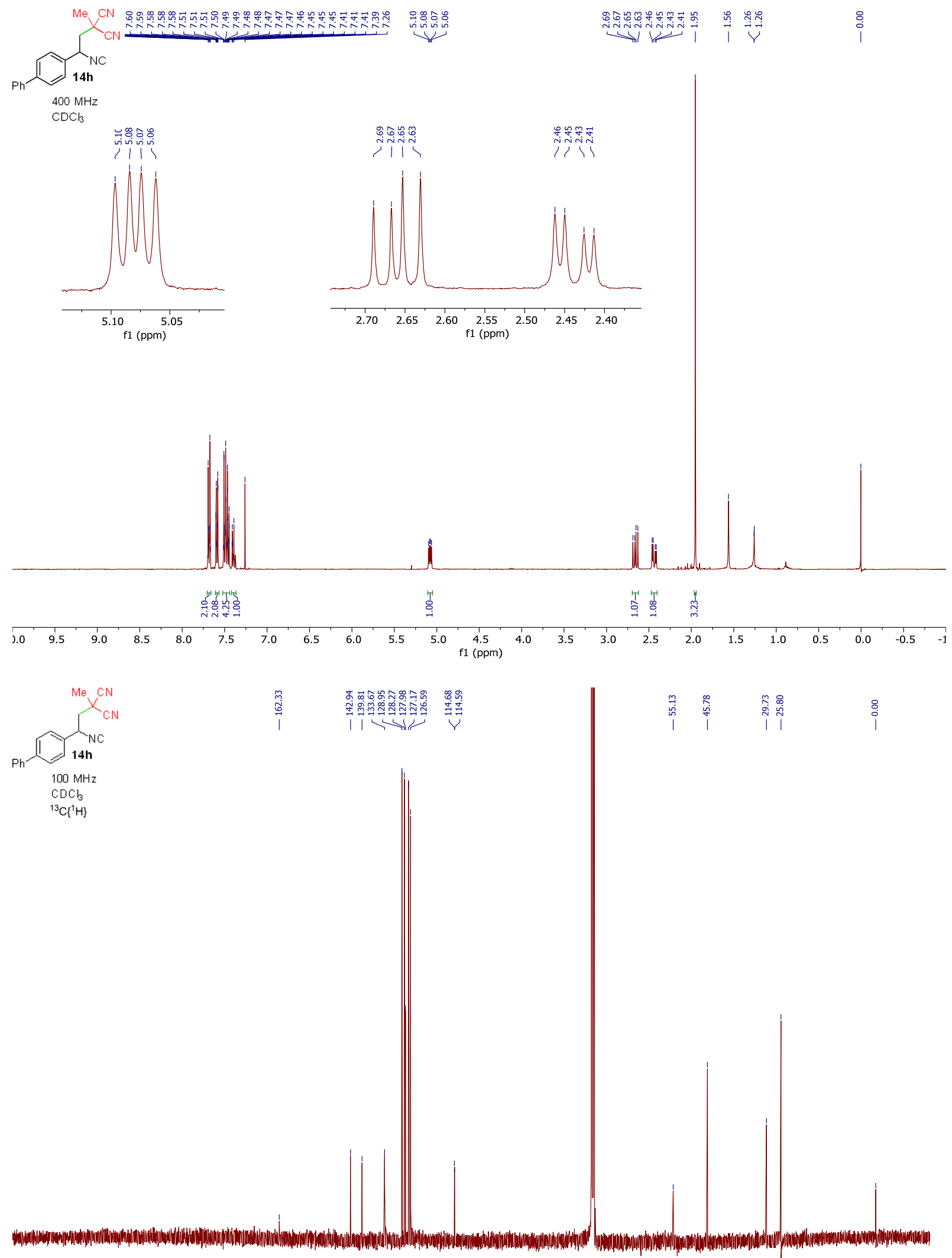

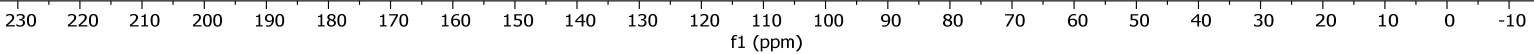



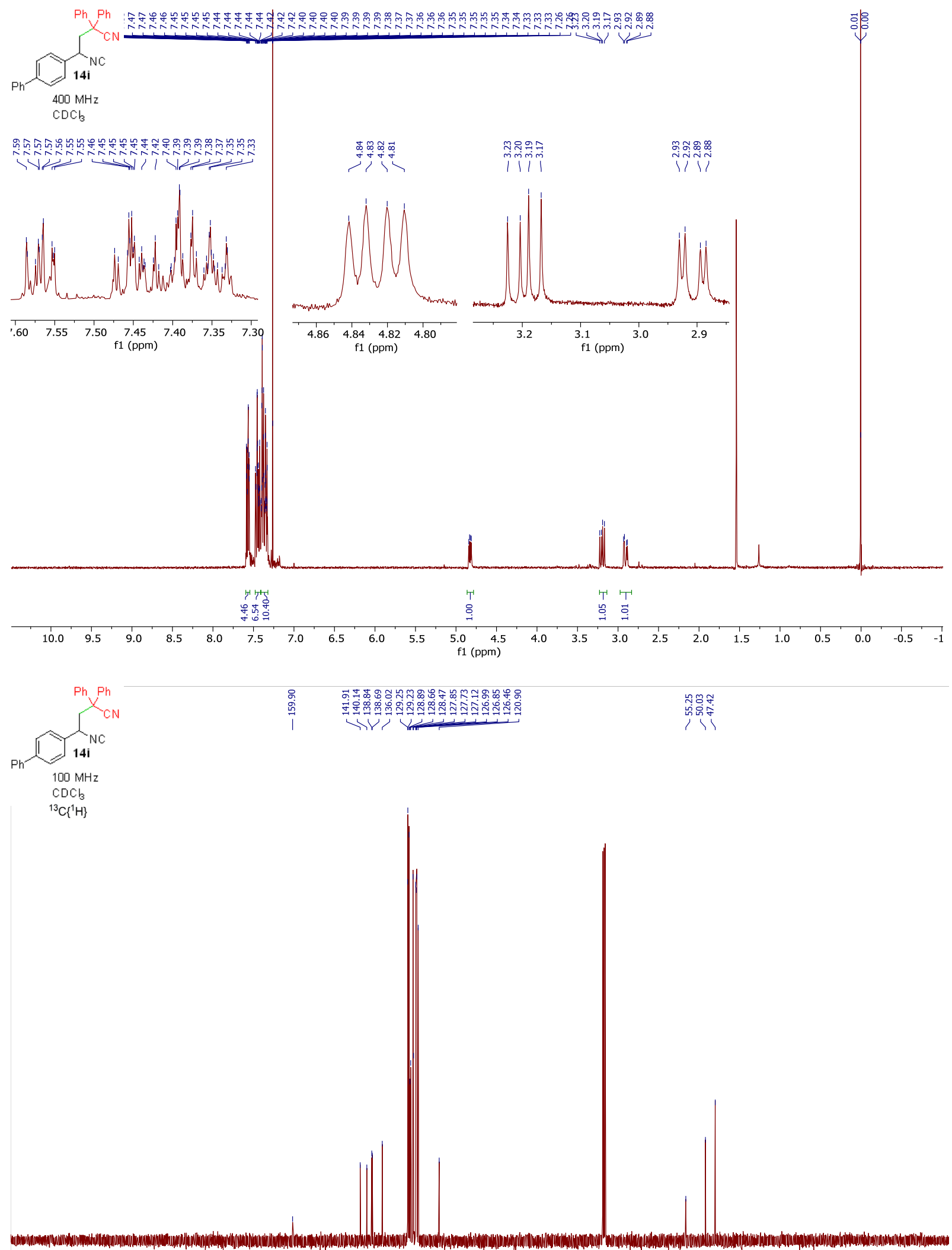

$\begin{array}{lllllllllllllllllllllllllllllllllll}230 & 220 & 210 & 200 & 190 & 180 & 170 & 160 & 150 & 140 & 130 & 120 & 110 & 100 & 90 & 80 & 70 & 60 & 50 & 40 & 30 & 20 & 10 & 0 & -10\end{array}$ 

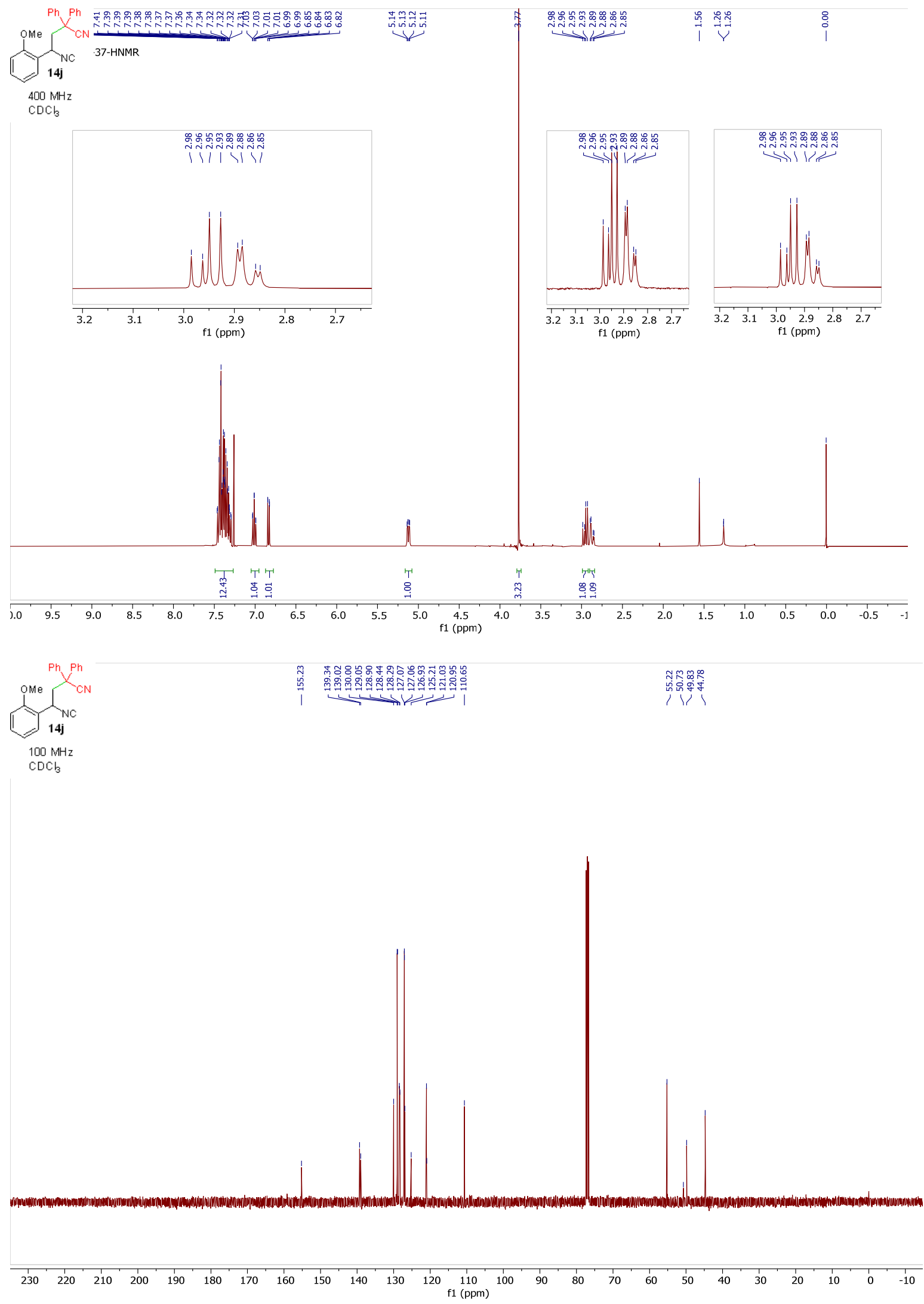

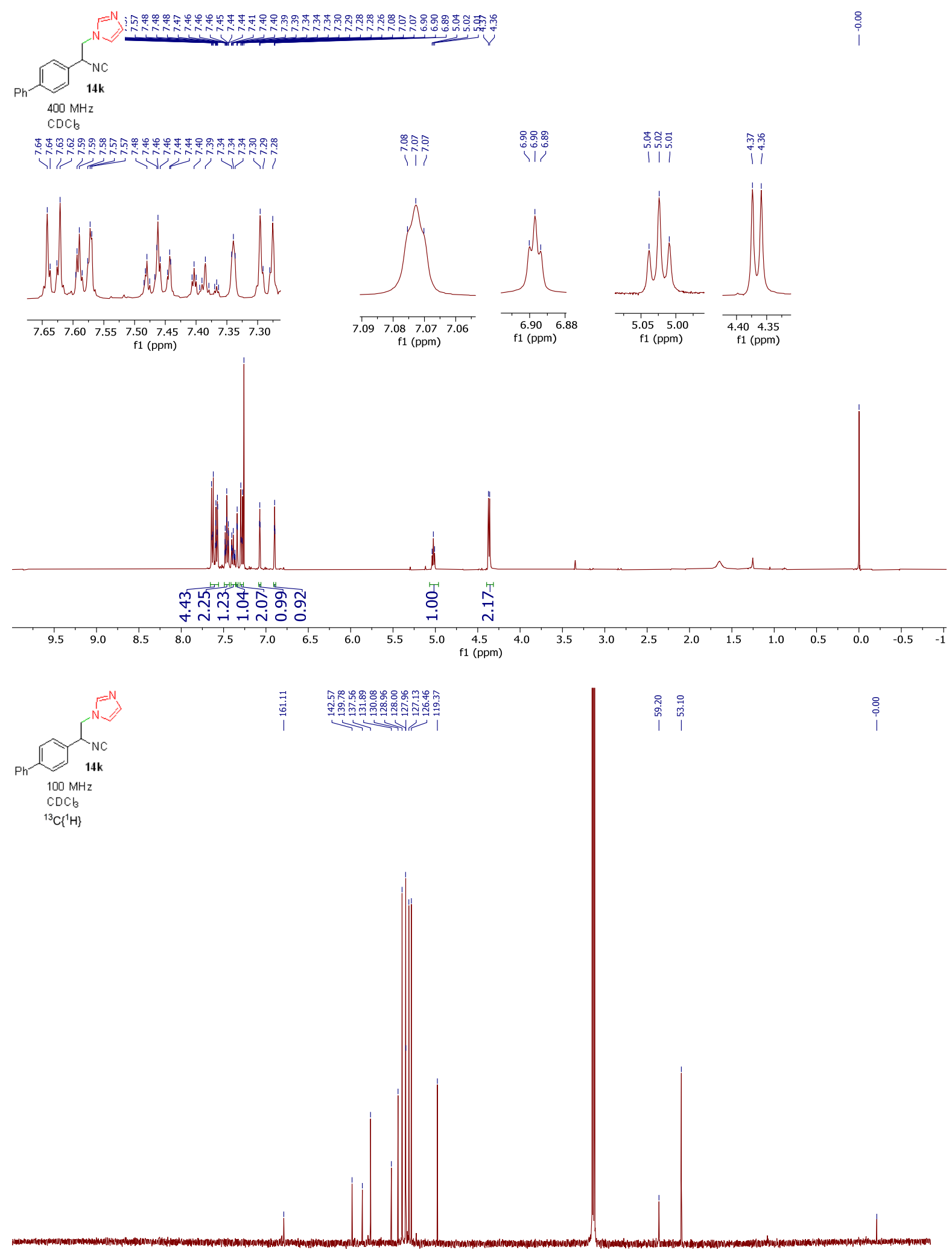

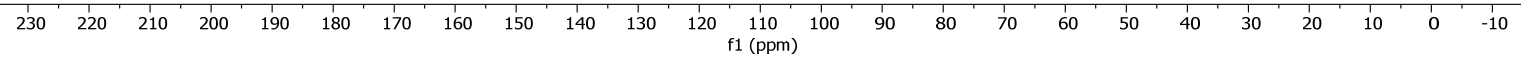



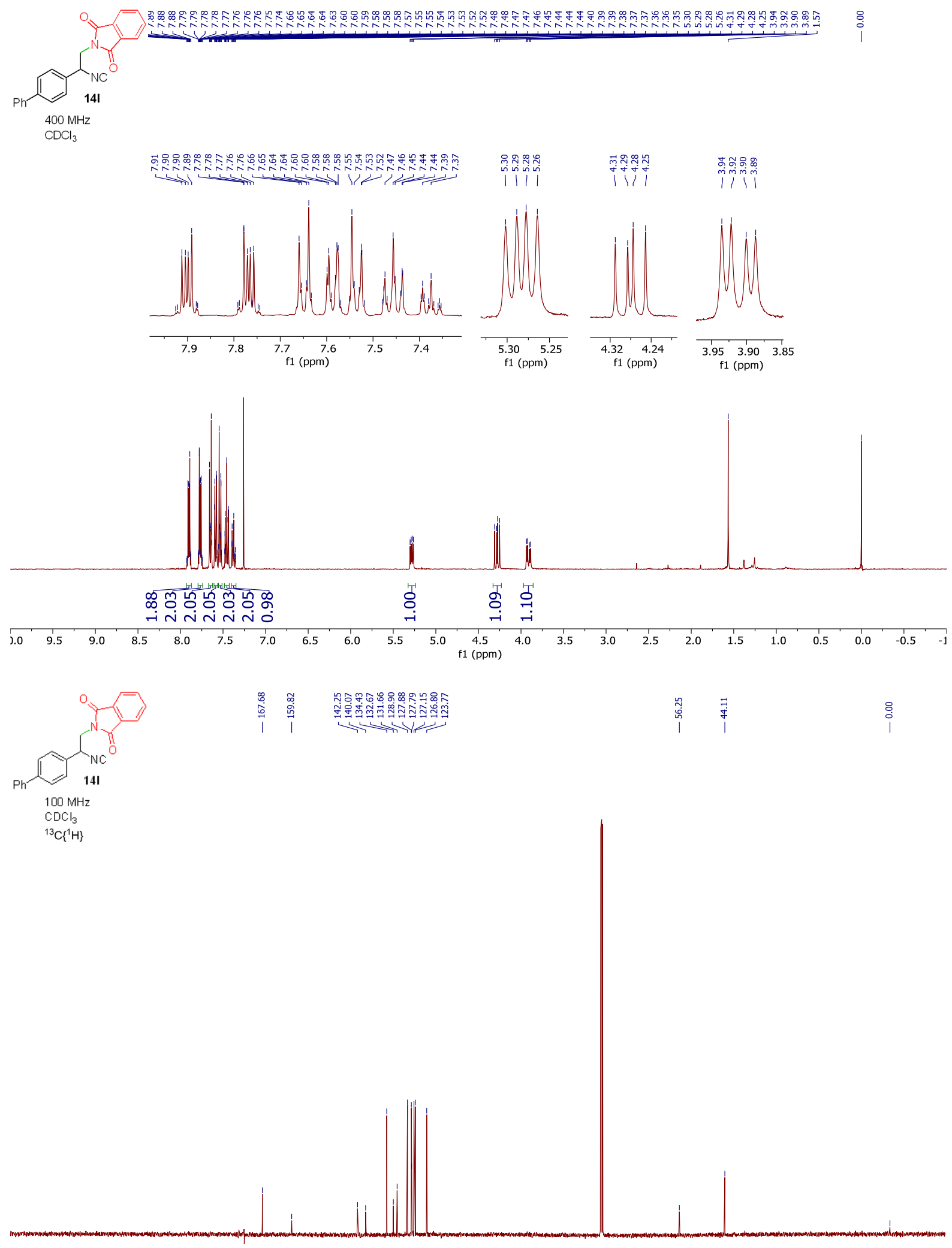

$\begin{array}{llllllllllllllllllllllllllllllll}230 & 220 & 210 & 200 & 190 & 180 & 170 & 160 & 150 & 140 & 130 & 120 & 110 & 100 & 90 & 80 & 70 & 60 & 50 & 40 & 30 & 20 & 10 & 0 & -10\end{array}$ 

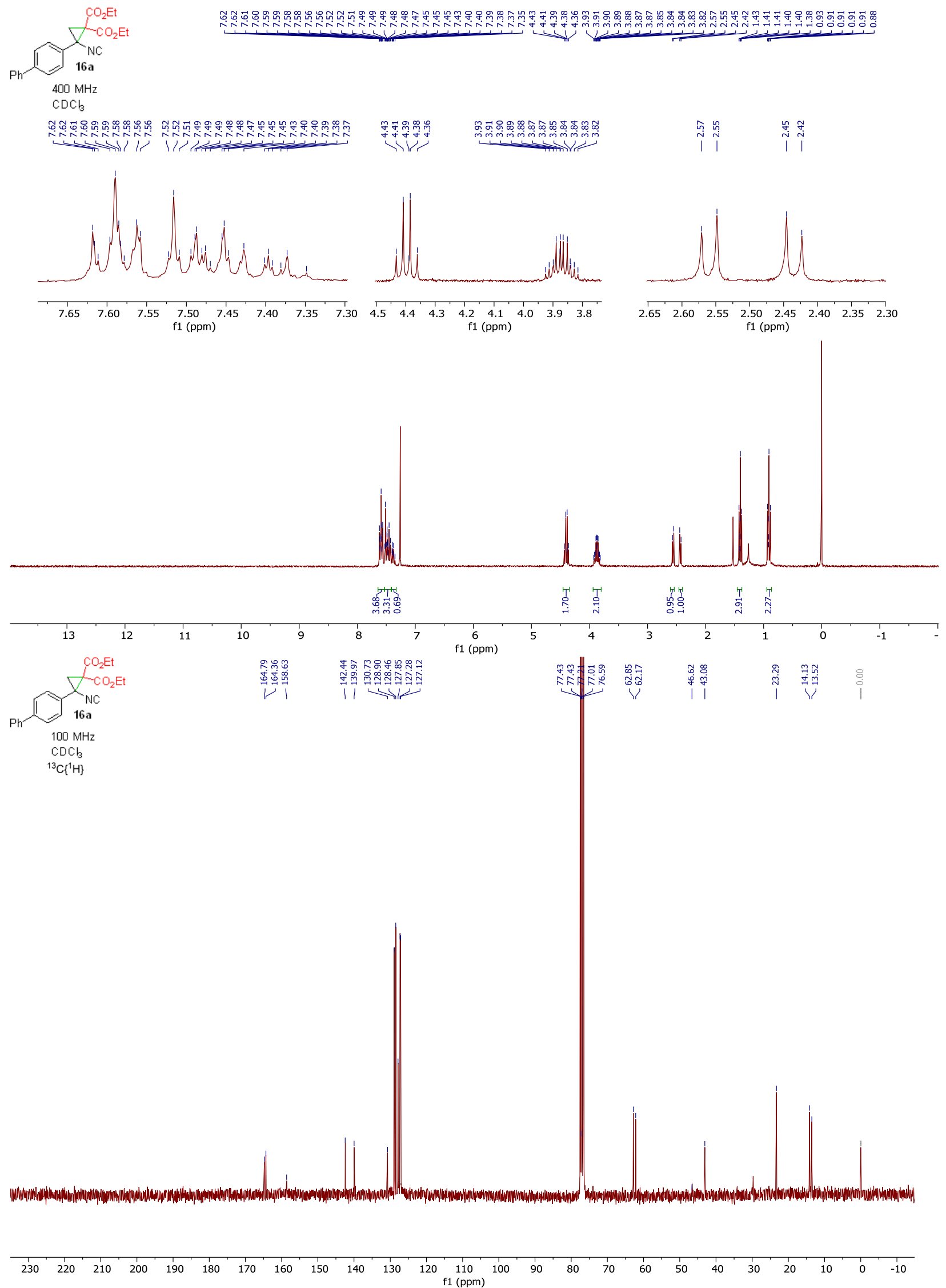


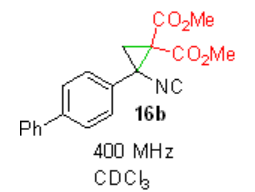

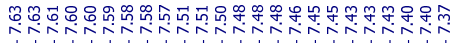

(1)

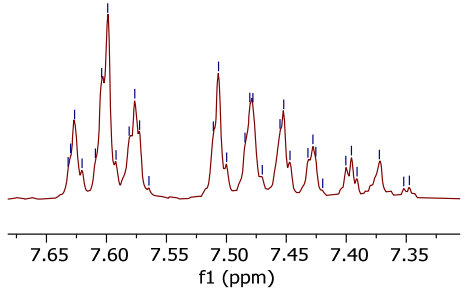

|

영ำ

Vil
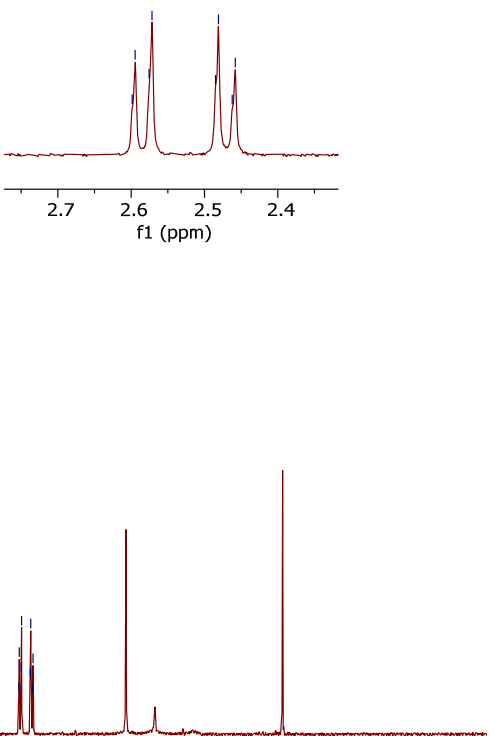

\begin{tabular}{llllllllllllllllll}
\hline 13 & 12 & 11 & 10 & 9 & 8 & 7 & 6 & 5 & 4 & 3 & 2 & 1 & 0 & -1 & 1 \\
\hline
\end{tabular}

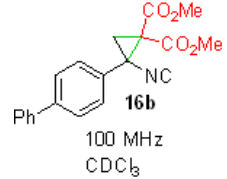

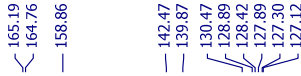

${ }^{13} \mathrm{C}\left\{{ }^{1} \mathrm{H}\right\}$

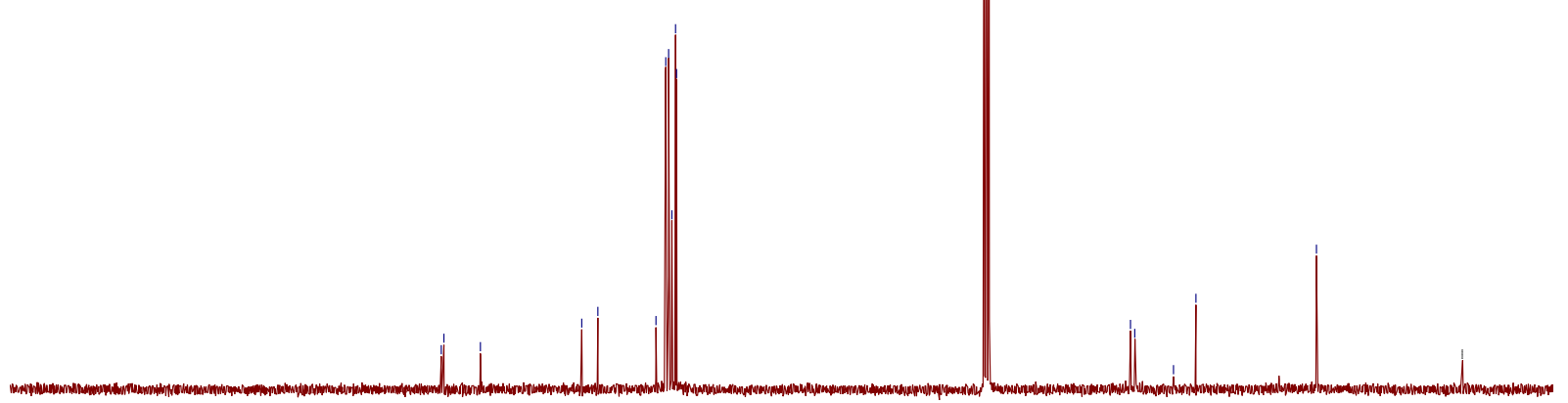

$\begin{array}{llllllllllllllllllllllllll}230 & 220 & 210 & 200 & 190 & 180 & 170 & 160 & 150 & 140 & 130 & 120 & 110 & 100 & 90 & 80 & 70 & 60 & 50 & 40 & 30 & 20 & 10 & 0 & -10\end{array}$ 


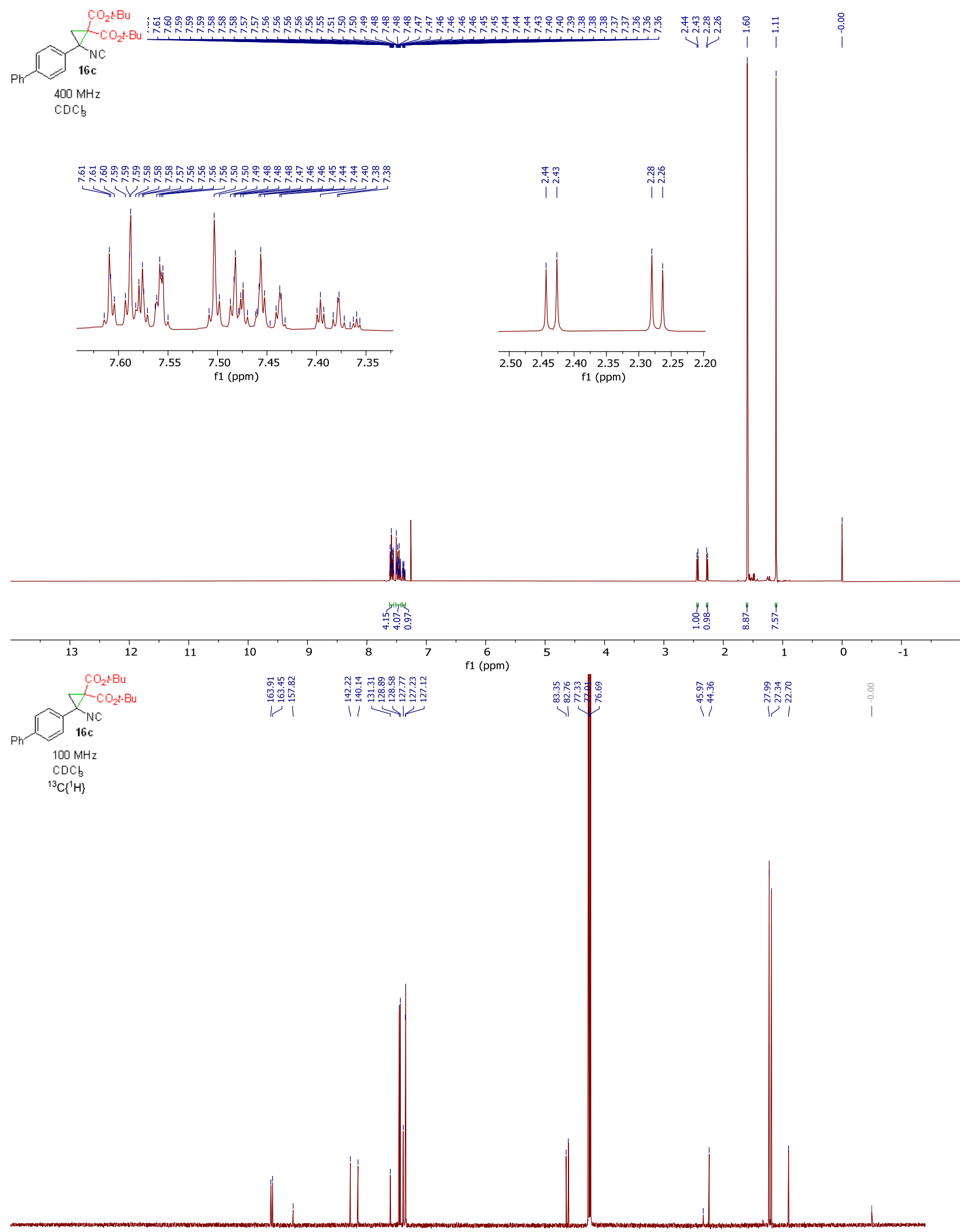

$\begin{array}{lllllllllllllllllllllllll}230 & 220 & 210 & 200 & 190 & 180 & 170 & 160 & 150 & 140 & 130 & 120 & \begin{array}{c}110 \\ \mathrm{f} 1(\mathrm{ppm})\end{array} & 100 & 90 & 80 & 70 & 60 & 50 & 40 & 30 & 20 & 10 & 0 & -10\end{array}$ 

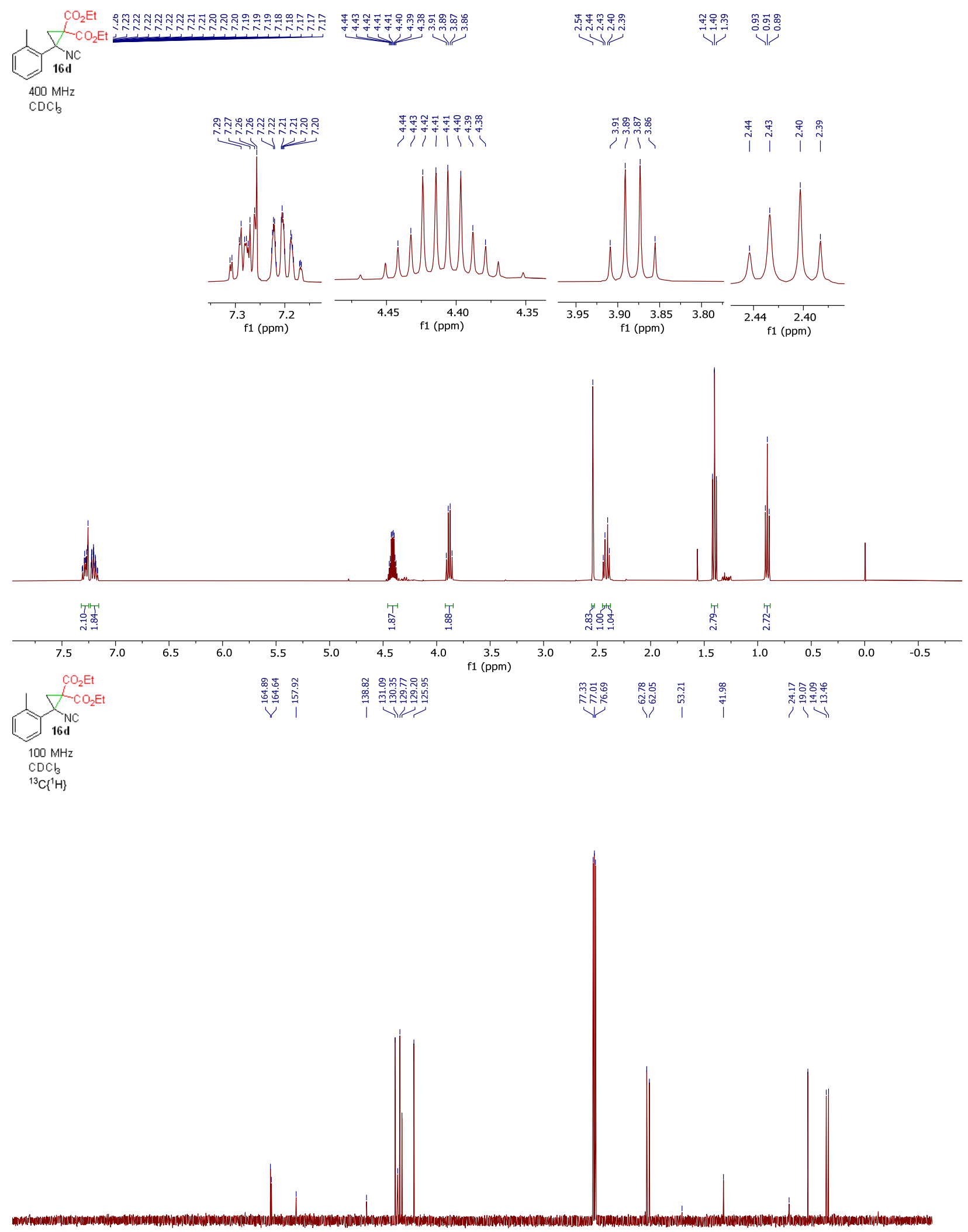

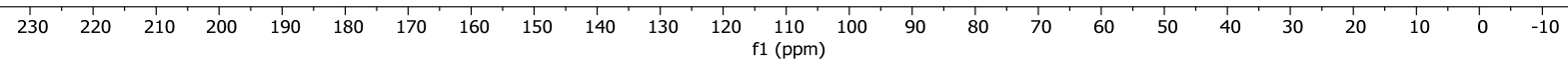



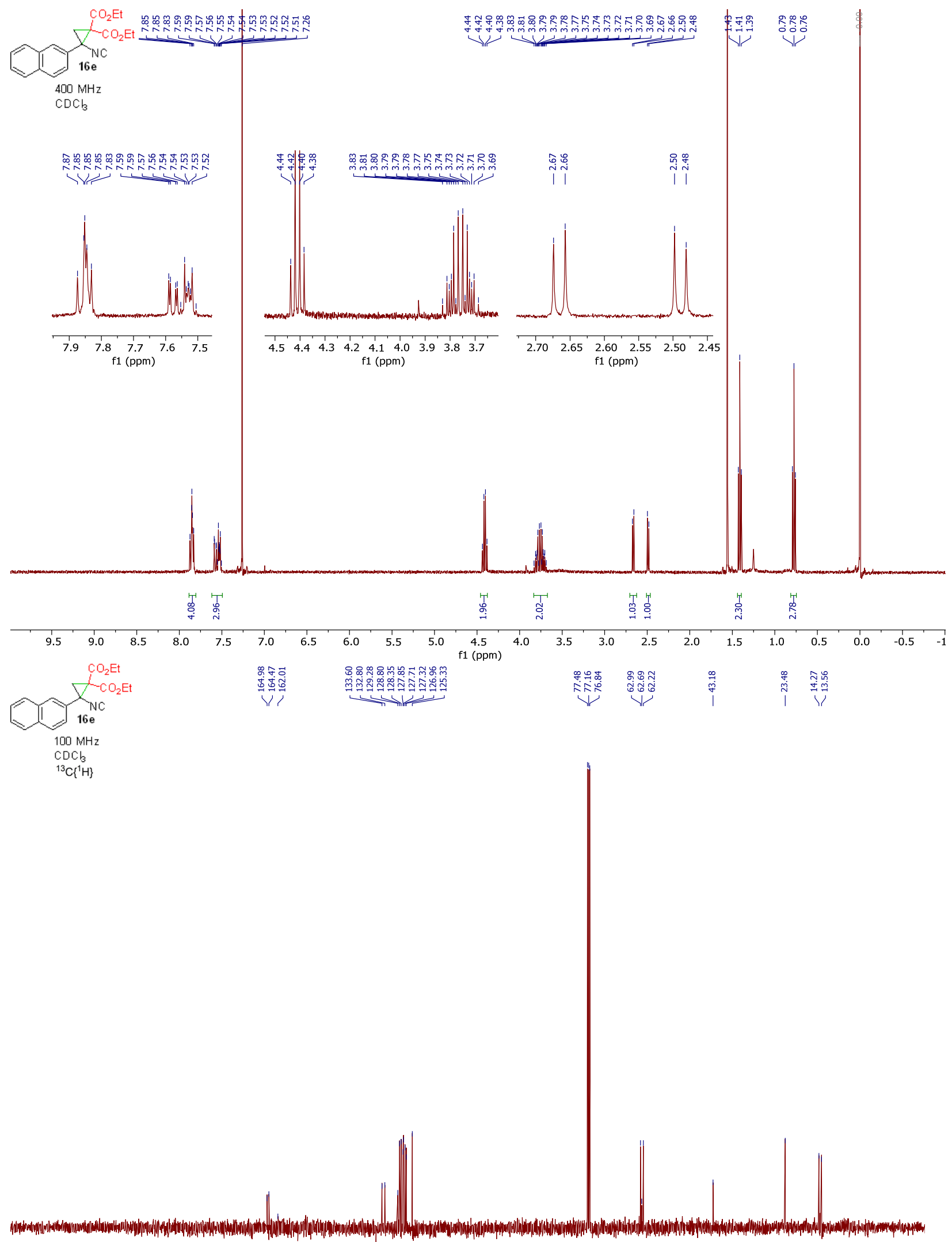

$\begin{array}{llllllllllllllllllllllllll}230 & 220 & 210 & 200 & 190 & 180 & 170 & 160 & 150 & 140 & 130 & 120 & 110 & 100 & 90 & 80 & 70 & 60 & 50 & 40 & 30 & 20 & 10 & 0 & -10\end{array}$ 

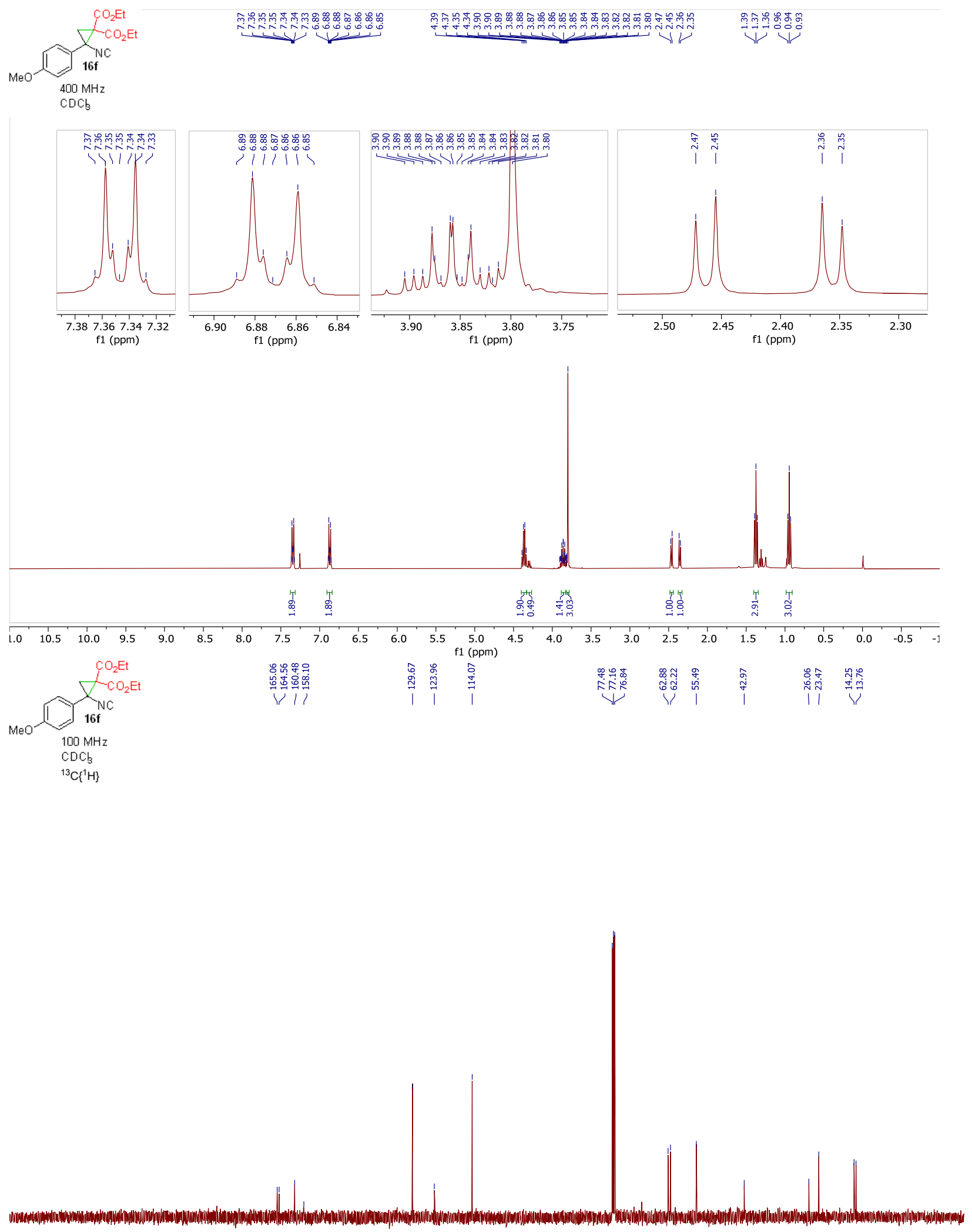

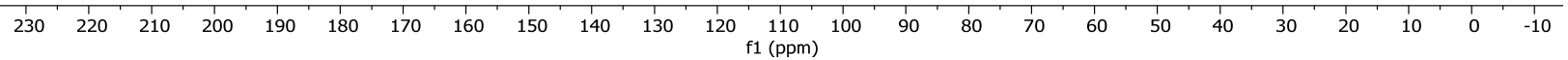



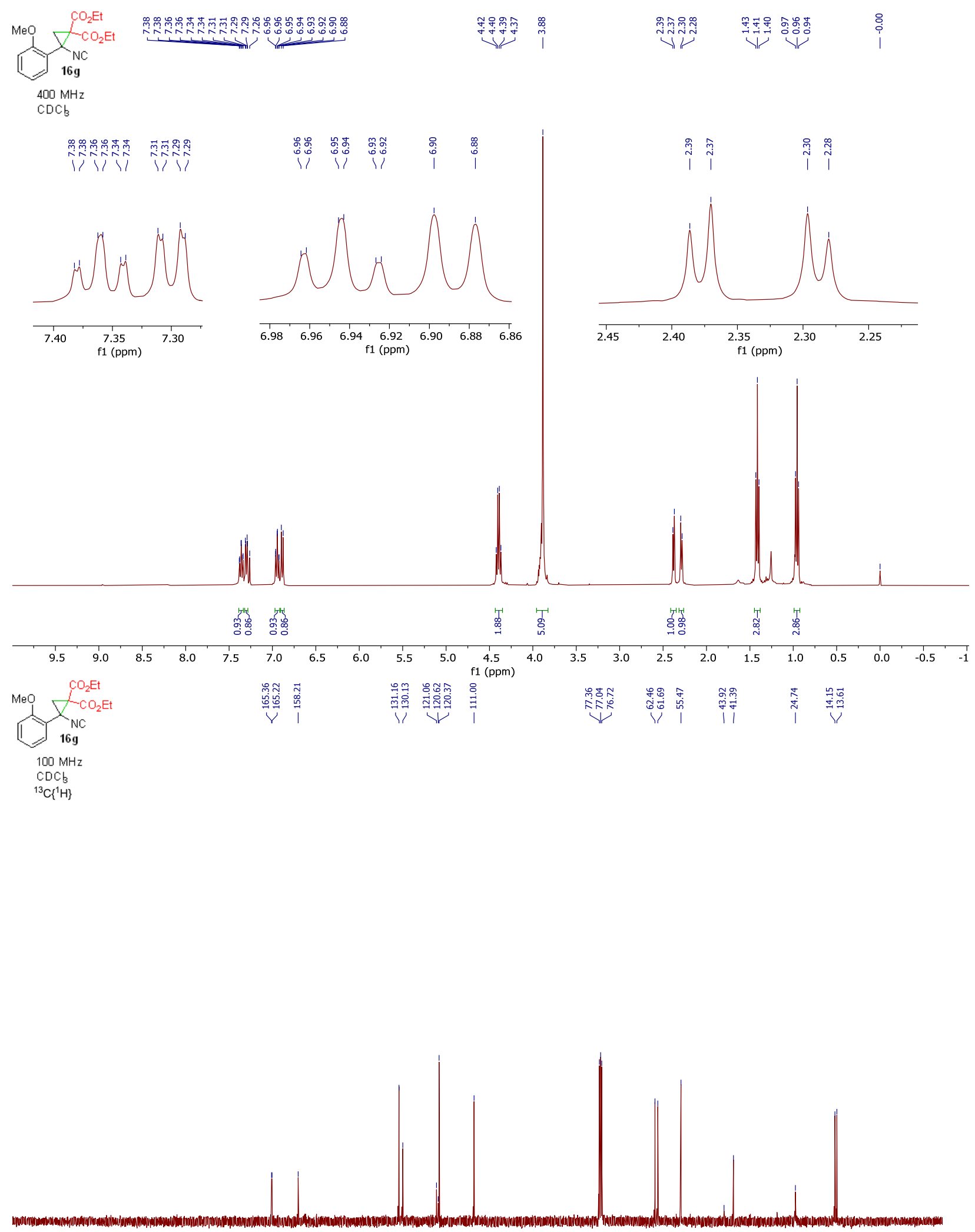

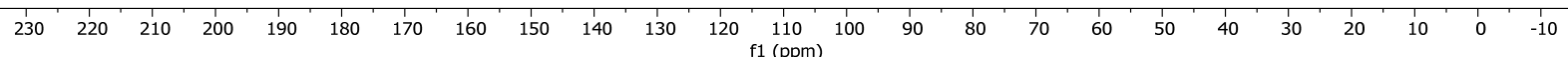



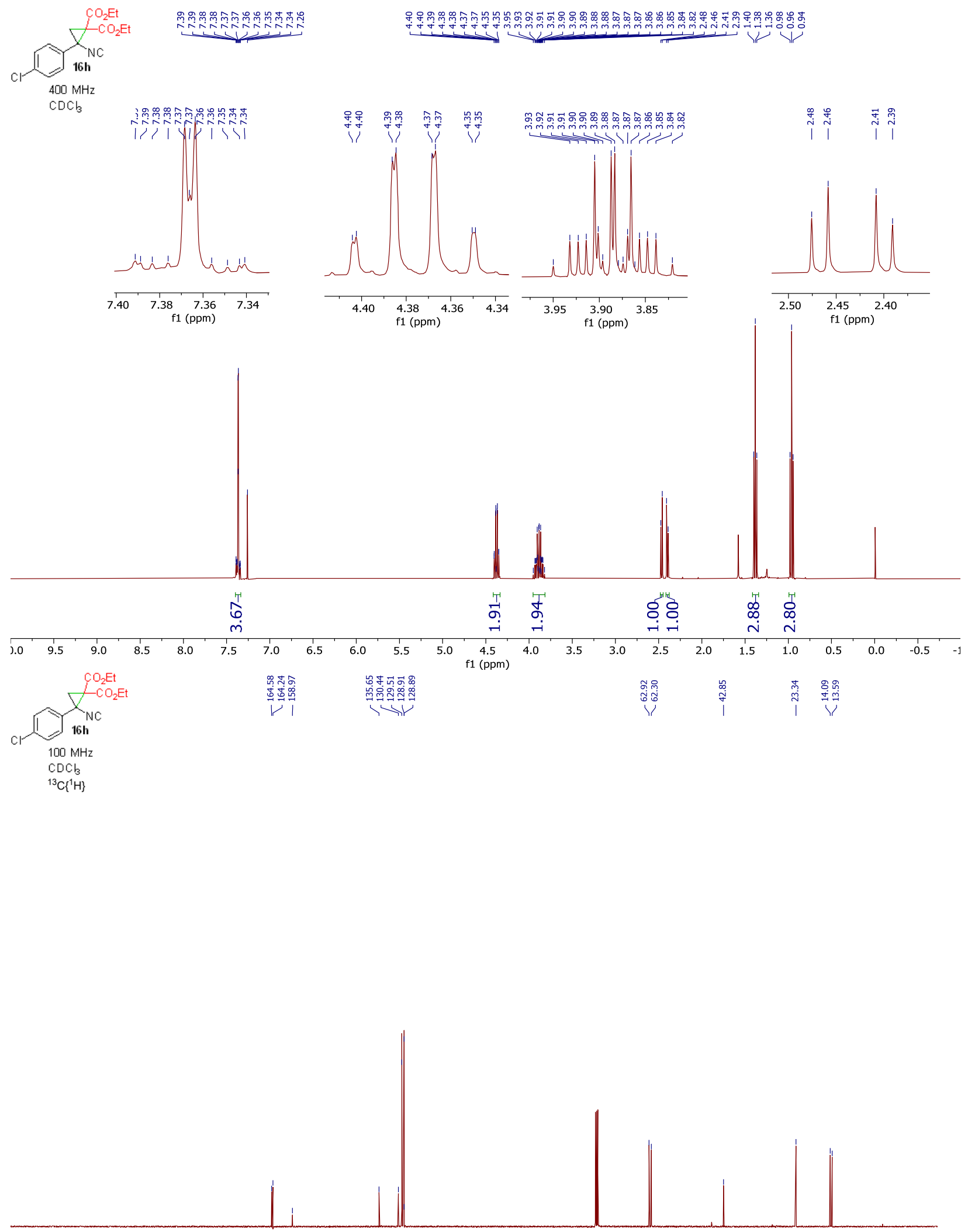

$\begin{array}{llllllllllllllllllllllllllll}230 & 220 & 210 & 200 & 190 & 180 & 170 & 160 & 150 & 140 & 130 & 120 & 110 & 100 & 90 & 80 & 70 & 60 & 50 & 40 & 30 & 20 & 10 & 0 & -10\end{array}$ 

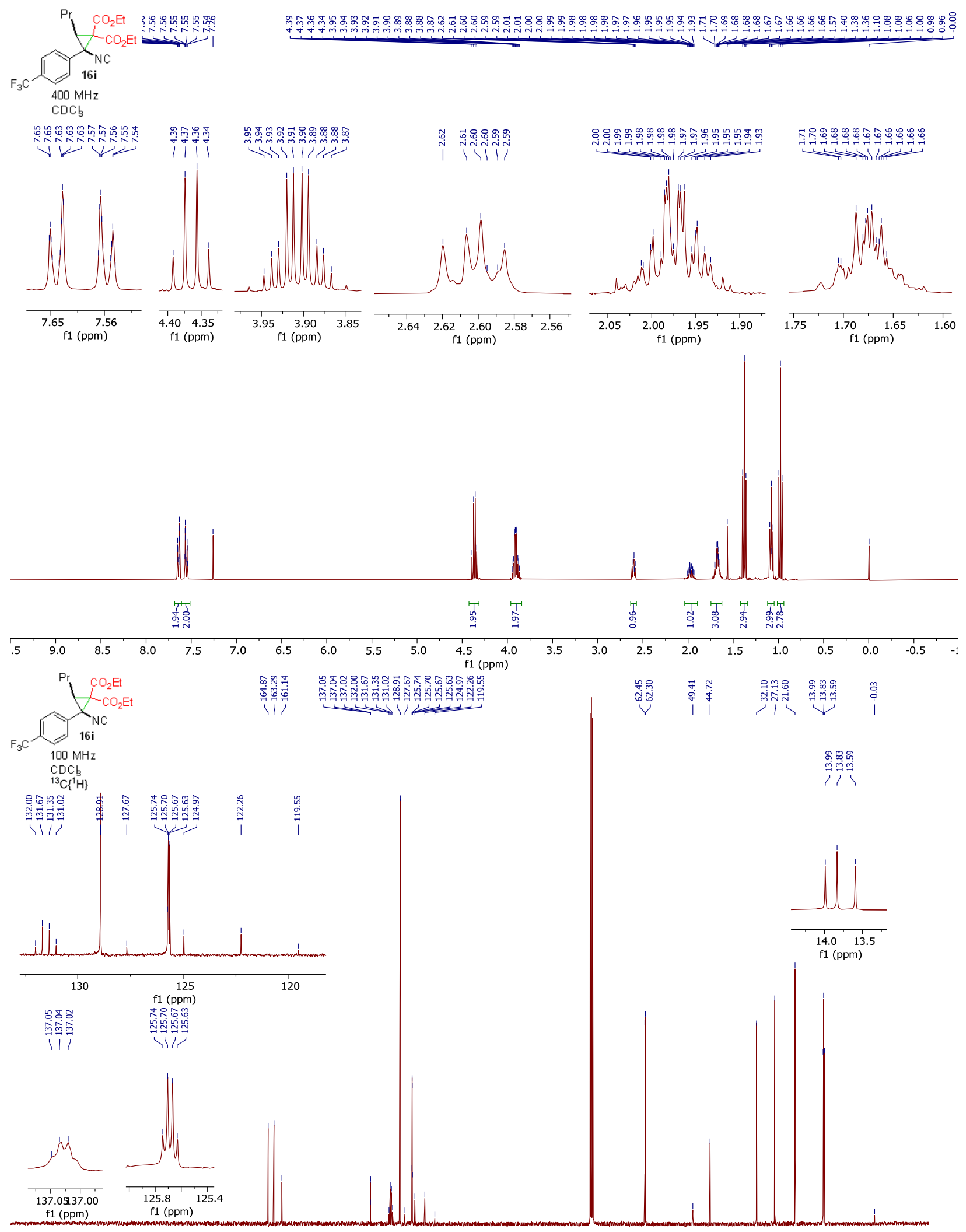

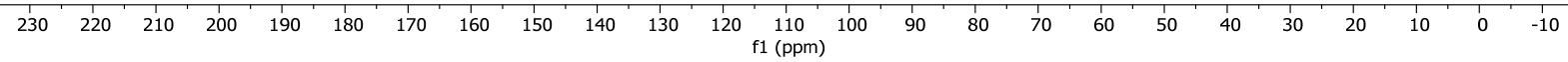



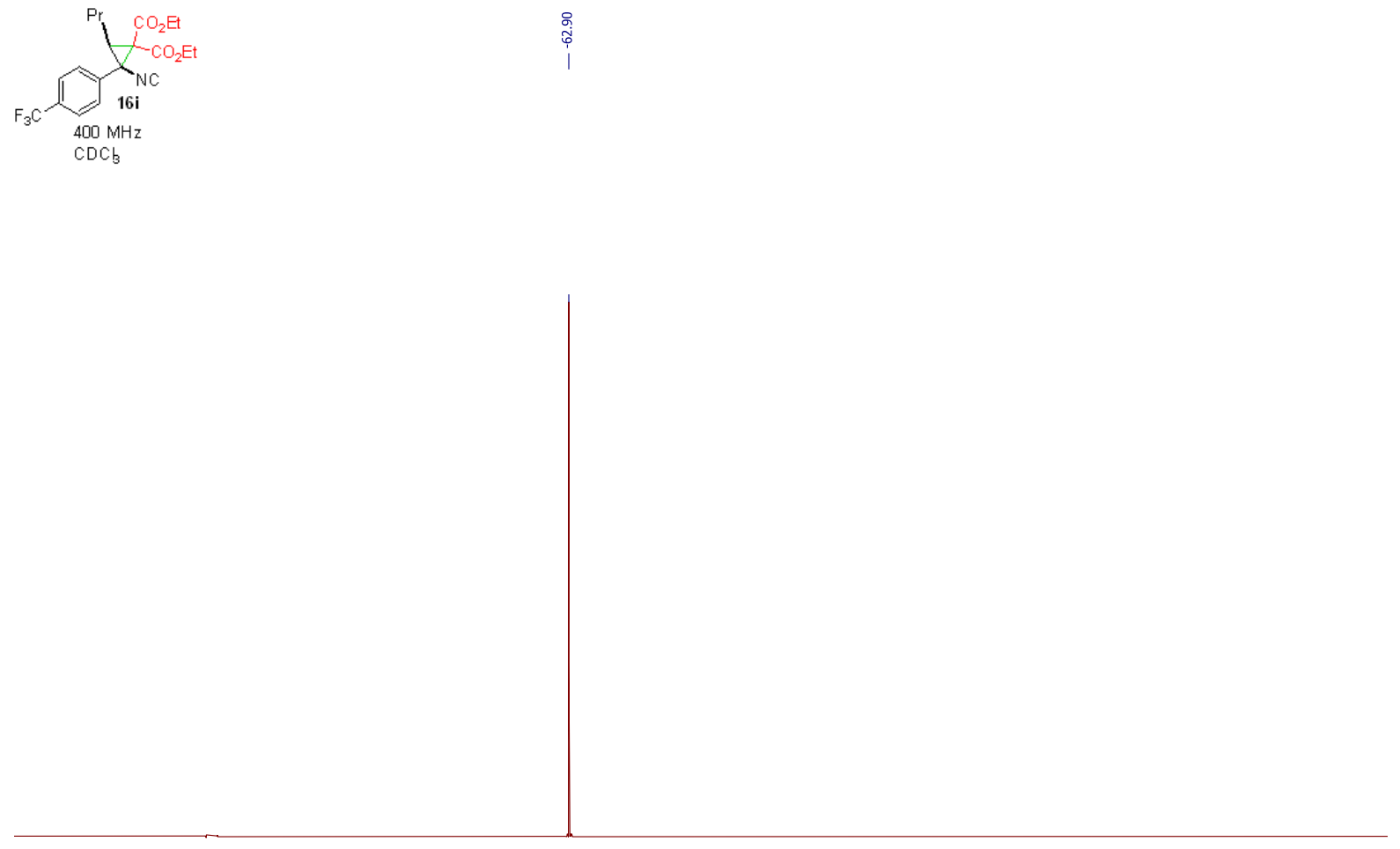

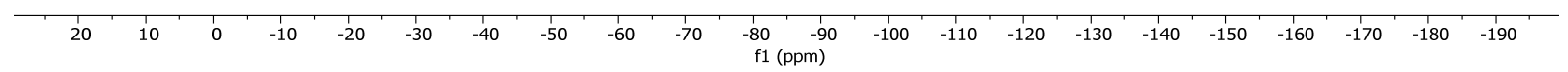

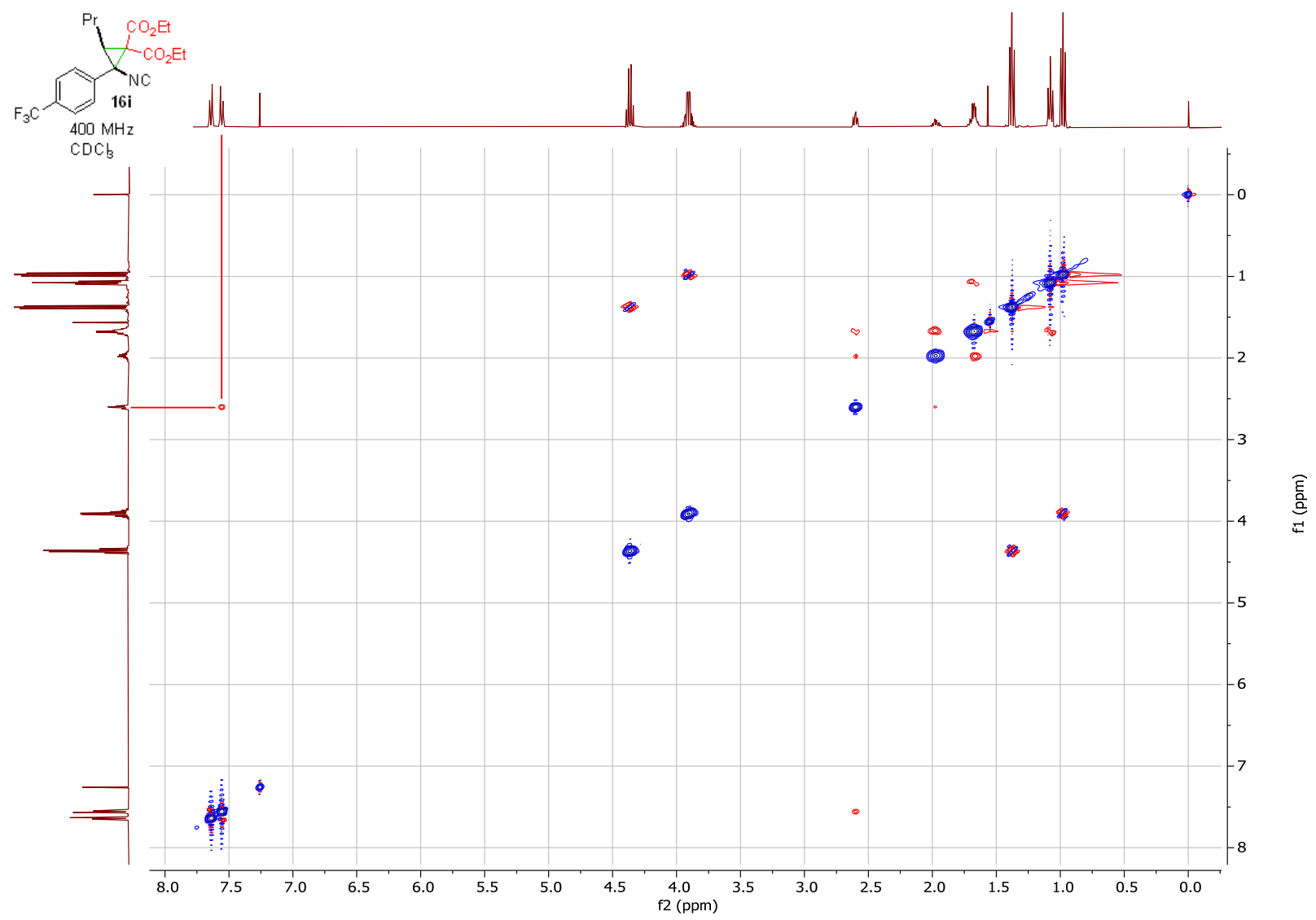



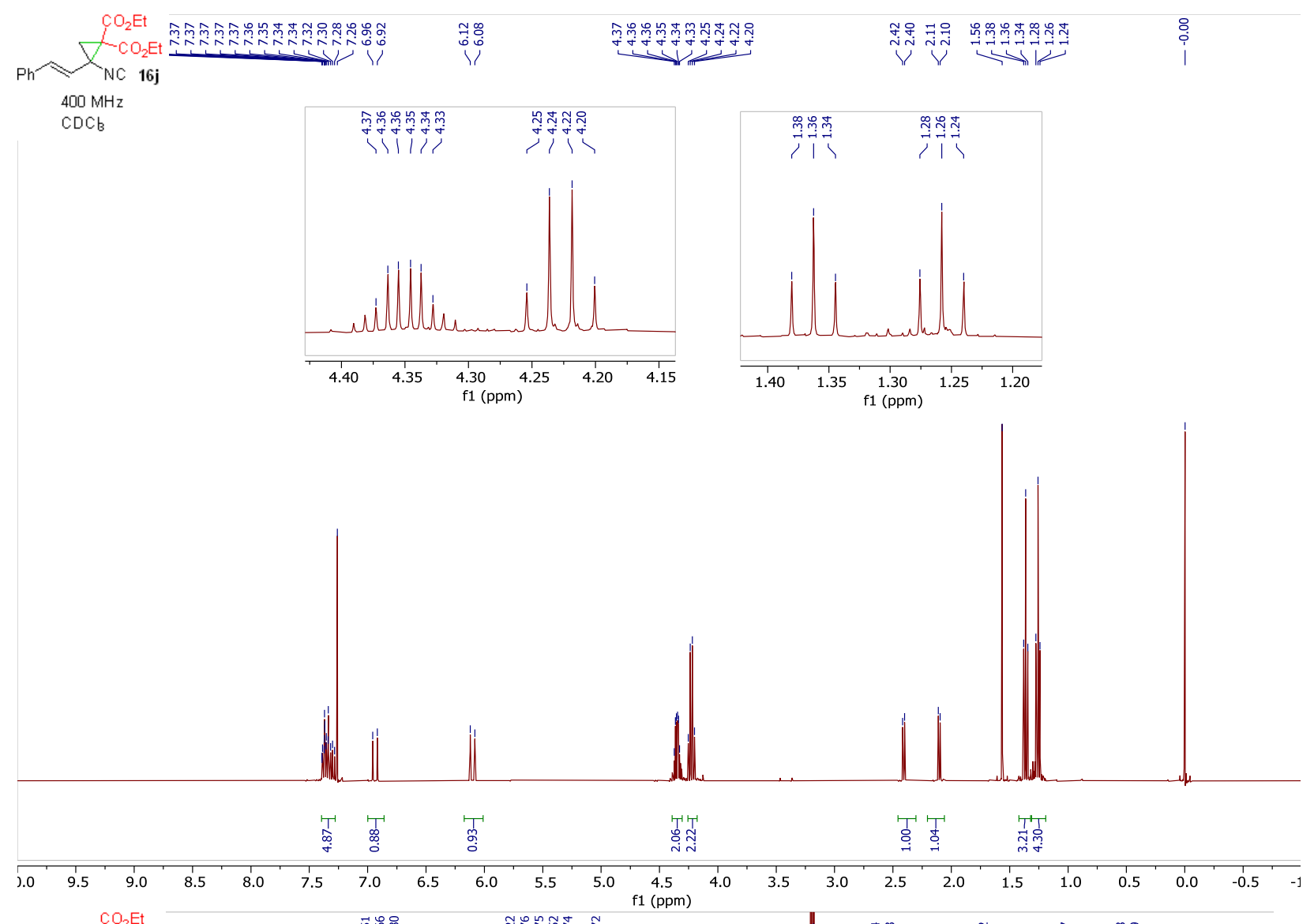

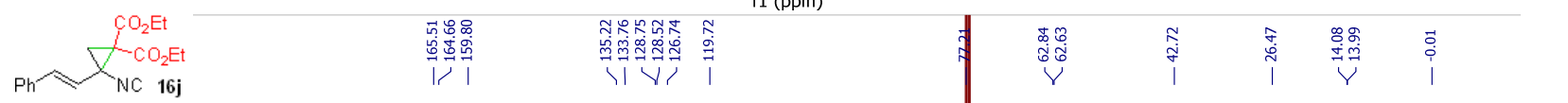
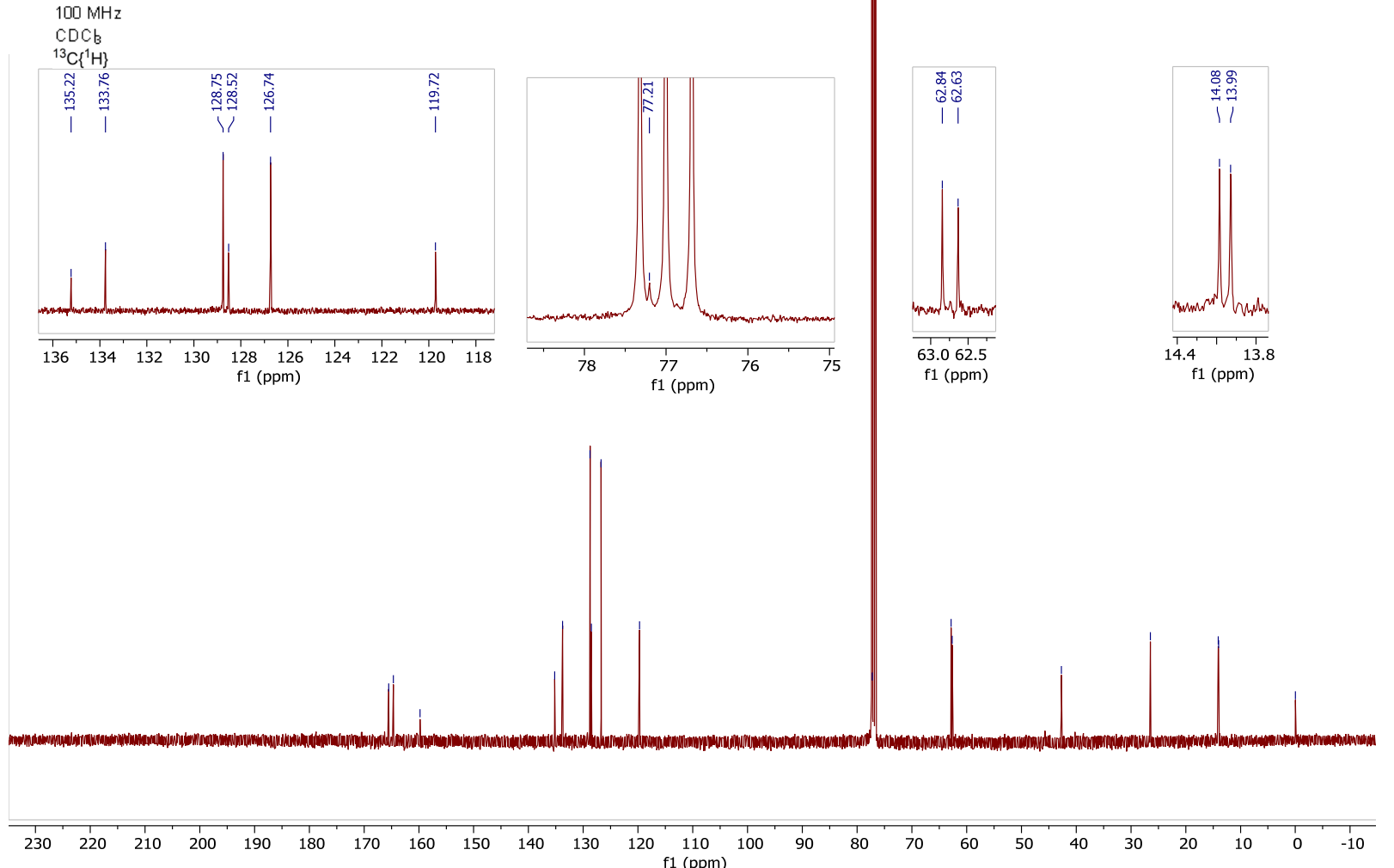


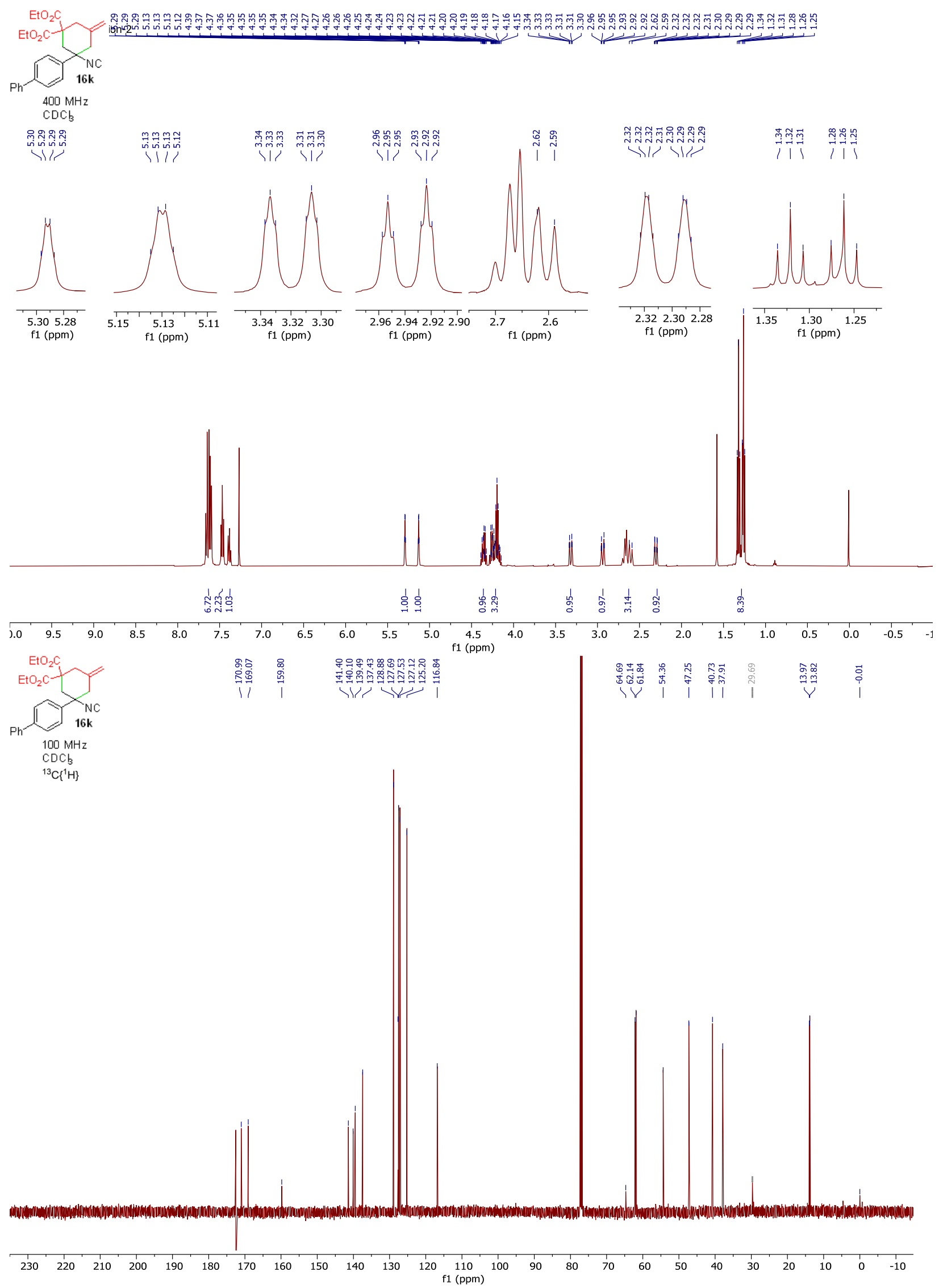




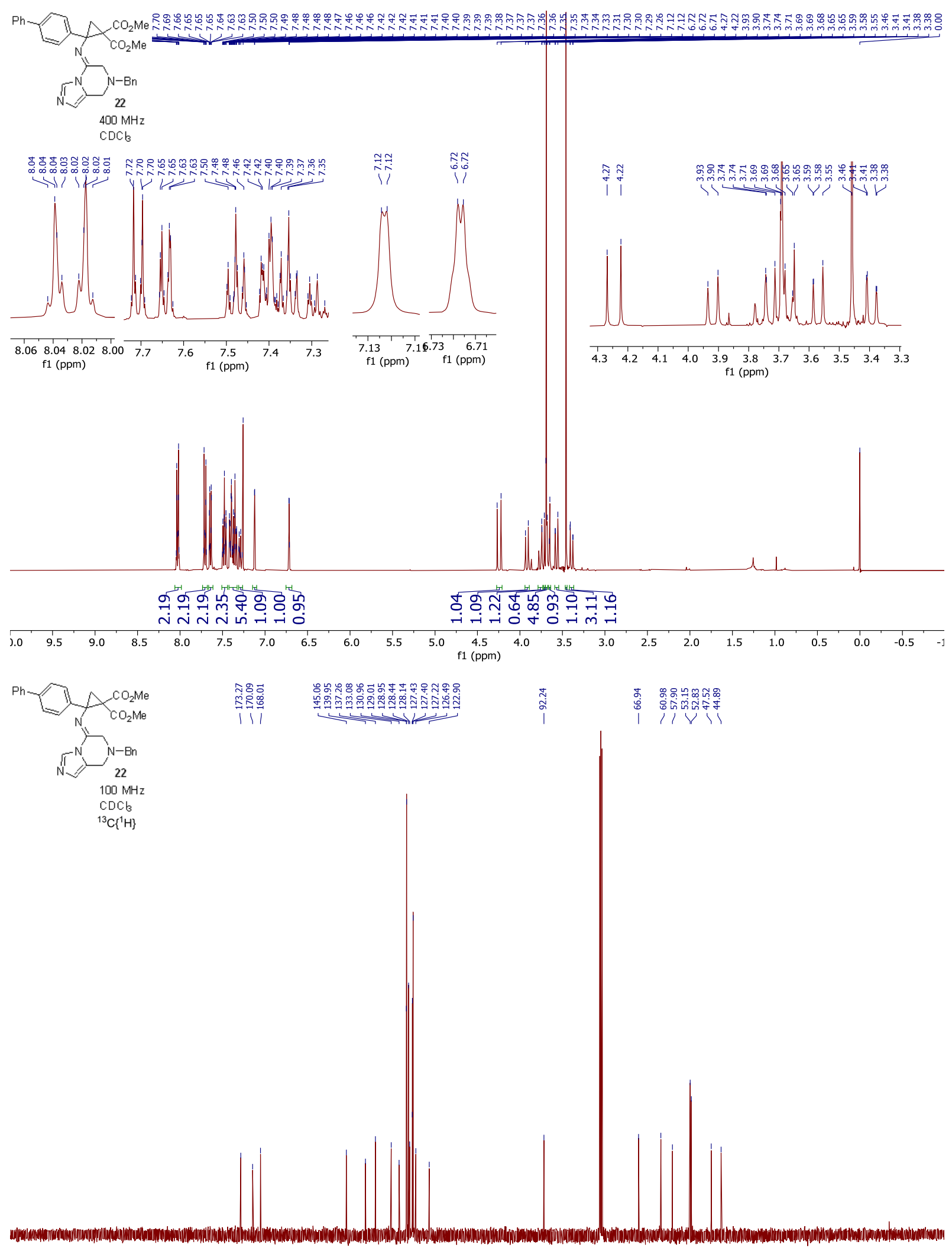

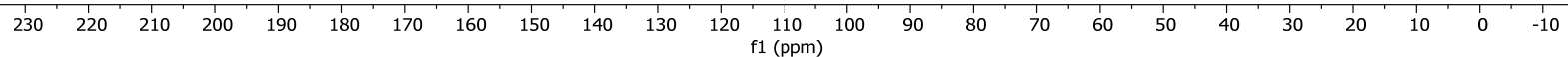


Figure S2. HPLC Chromatogram of $\mathbf{1 6 b}$

\section{- simmazu Analysis Report}

<Sample Information>

Sample Name : JP-methyl-cyclopropane

Sample ID

Data Filename : JP-methyl-cyclopropane6.Icd

Method Filename : test2.lcm

Batch Filen

Vial \# $: 1-6$

Injection Volume : $5 \mathrm{uL}$

Date Acquired $\quad: 8 / 17 / 20211: 50: 11 \mathrm{PM}$

Date Processed $\quad: 8 / 17 / 20212: 05: 48$ PM

Sample Type : Unknown

Acquired by : System Administrator

Processed by : System Administrator

\section{$<$ Chromatogram>}

$\mathrm{mAU}$

Chromatogram

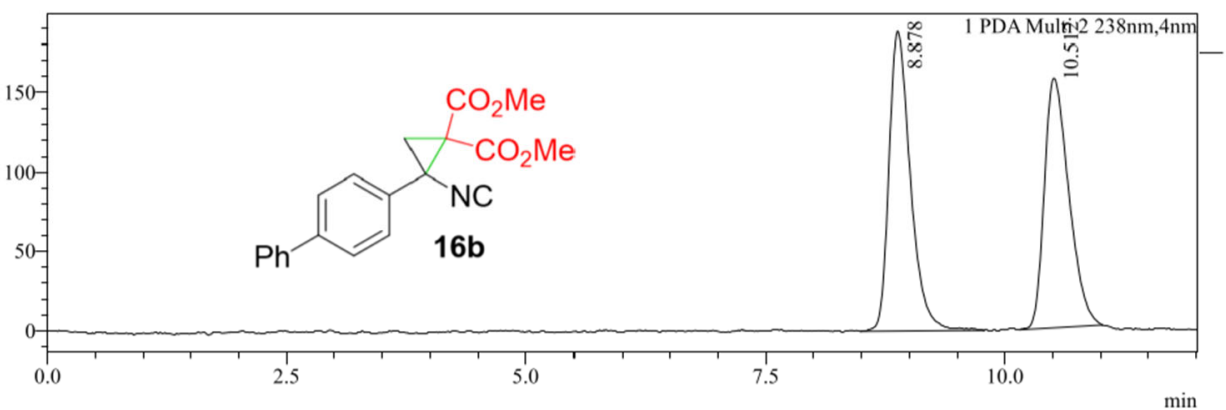

<Peak Table>

PDA Ch2 238nm

\begin{tabular}{|r|r|r|r|r|r|r|r|}
\hline Peak\# Ret. Time & Area & Height & \multicolumn{1}{|c|}{ Conc. } & Unit & Mark & Name \\
\hline 1 & 8.878 & 2948761 & 187872 & 51.289 & & S & \\
\hline 2 & 10.512 & 2800520 & 156855 & 48.711 & & & \\
\hline Total & & 5749282 & 344727 & & & &
\end{tabular}




\section{Figure S3. Suppression of downfield ${ }^{13} \mathrm{C}$ NMR Resonances on Addition of $\mathrm{Cu}(\mathrm{I}) \mathrm{I}$ to $\mathbf{1 3 a}$.}

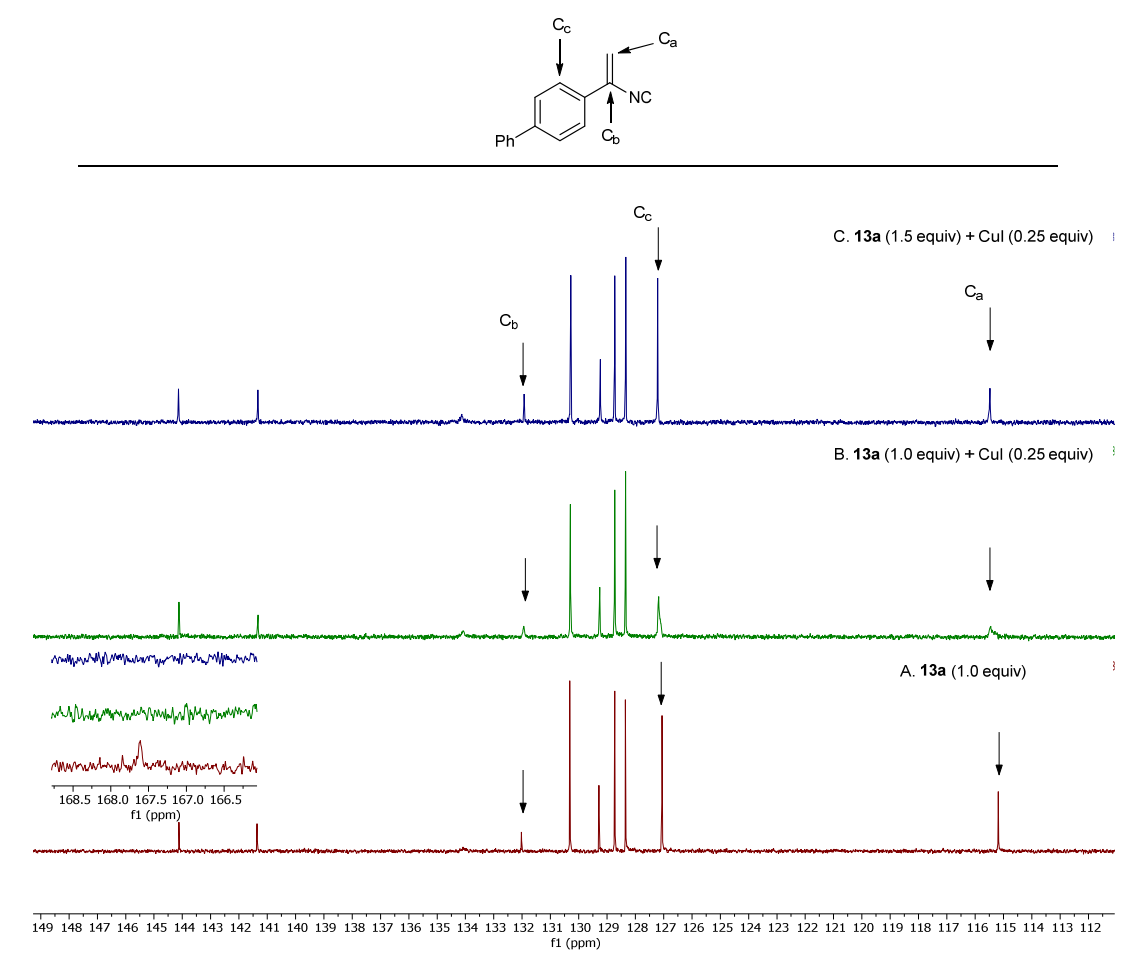

${ }^{13} \mathrm{C}$ NMR was used to probe the complexation of $\mathrm{Cu}(\mathrm{I}) \mathrm{I}$ with the alkeneisocyanide $13 \mathbf{a} .{ }^{1}$ Addition of 0.25 equivalents of $\mathrm{Cu}(\mathrm{I}) \mathrm{I}$, sufficient for coordinative saturation, to a $\mathrm{d}_{8}$-THF solution of alkeneisocyanide 13a (Figure 2, A) suppressed the ${ }^{13} \mathrm{C}$ NMR isocyanide signal and attenuated the signals from the olefinic carbon (115 ppm), the proximal quaternary carbon (132 ppm), and ortho-methine signals (127, $130 \mathrm{ppm}$ ) (Figure 2, B). Addition of 0.5 equivalents of 13a to the solution restored the amplitude of the olefin and phenyl carbon signals, though the isocyanide signal was noticeably absent (Figure 2, C). Interestingly, the signals appear to be most similar to the free isocyanide as opposed to a composite of free and complexed isocyanides. The signal intensities are consistent with a fast, reversible complexation of $\mathrm{Cu}(\mathrm{I}) \mathrm{I}$ with the isocyanides. ${ }^{2}$ 


\section{References}

1 Knol, D.; Koole, N. J.; de Bie, M. J. A. A 13C NMR Investigation of some Tetrakisisocyanocopper(1)

Tetrafluoroborate Complexes: Chemical Shift of, and Coupling to, the Isocyano Carbon Org. Magn. Reason. 1976, 8, 213-218.

2 Pintauer, T. Towards the development of highly active copper catalysts for atom transfer radical addition (ATRA) and polymerization (ATRP) Chem. Pap. - Chem. Zvesti 2016, 70, 22-42. 